\title{
ARE SUNSCREENS A NEW ENVIRONMENTAL RISK ASSOCIATED TO COASTAL TOURISM?
}

David Sánchez-Quiles*a ${ }^{* a}$ Antonio Tovar-Sánchez ${ }^{\mathrm{a}, \mathrm{b}}$

a Department of Global Change Research. Mediterranean Institute for Advanced Studies, IMEDEA (CSIC-UIB), Miguel Marqués 21, 07190 Esporles, Balearic Island, Spain.

${ }^{b}$ Department of Ecology and Coastal Management. Andalusian Institute for Marine Science, ICMAN (CSIC). Campus Universitario Río San Pedro, 11510 Puerto Real, Cádiz. Spain.

\section{Corresponding Author}

*David Sánchez-Quiles. Department of Global Change Research. Mediterranean Institute for Advanced Studies, IMEDEA (CSIC-UIB), Miguel Marqués 21, 07190 Esporles, Balearic Island, Spain. Telephone: +34 971611726. E-mail: dsanchez@imedea.uib-csic.es. 


\begin{abstract}
The world coastal-zone population and the coastal tourism are expected to grow during this century. Associated to that, there is an increase in the use of sunscreens, and cosmetics with UV-filters in their formulation, that point out coastal regions worldwide susceptible to receive the impact of these cosmetics. Recent investigations indicate that organic UV-filters and inorganic oxide nanoparticles as well as many other components that are constituents of the sunscreens reach the marine environment and cause harmful effects. This review examines the research studies done on sunscreens in the environment, highlights the environmental risk in coastal areas and suggests future directions.
\end{abstract}

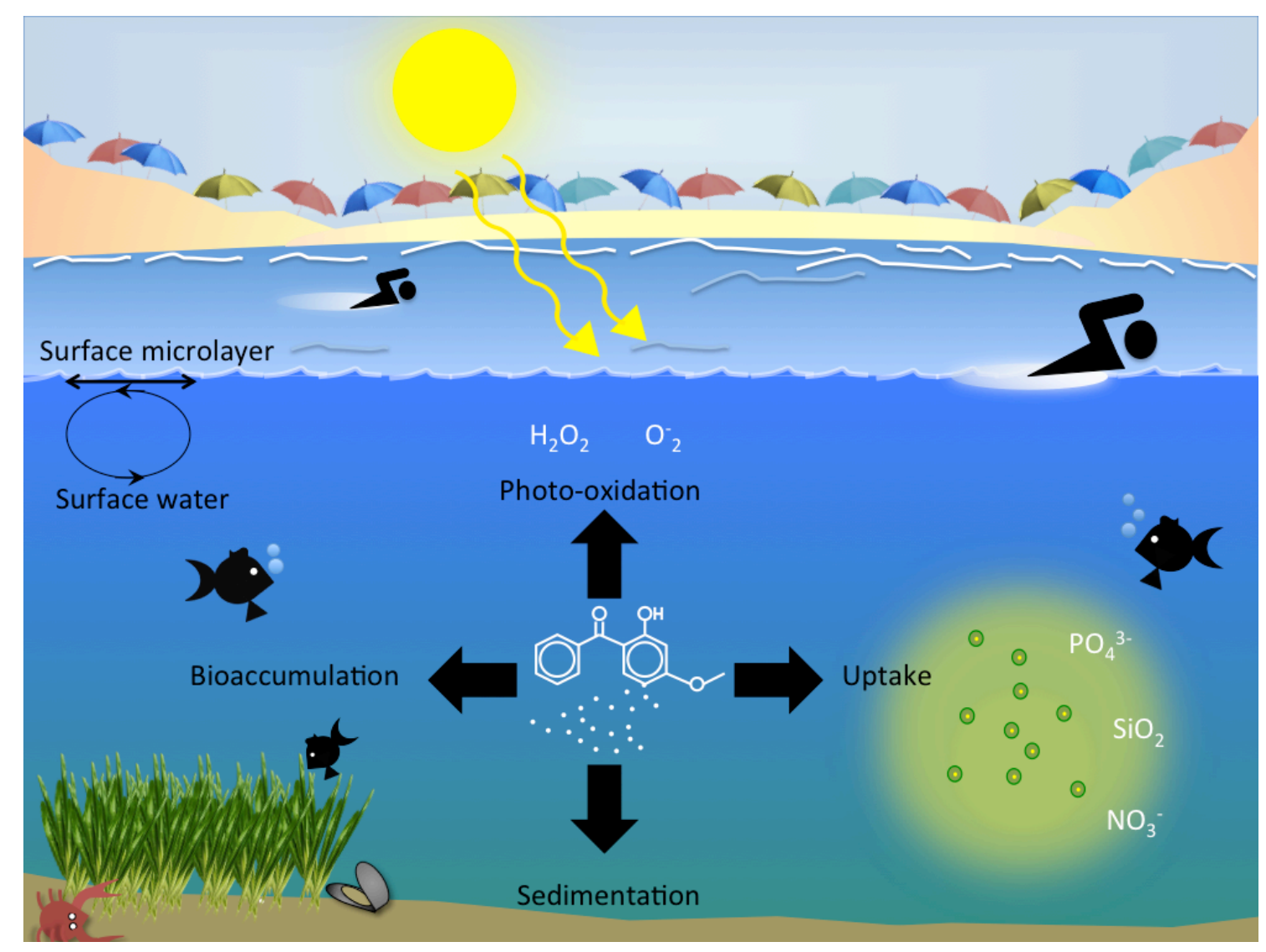

Keywords: Sunscreens; UV-filters; nanoparticles; coastal tourism; environmental risk; marine pollution. 


\section{Highlight:}

1. Revision of research studies on sunscreens in the environment.

2. Environmental implications of sunscreen's components into the coastal ecosystem.

3. More studies about sunscreens in the environment need to be addressed. 


\section{Introduction}

Throughout the history of human beings, the sun has been an object of adoration and exploration. However, it was not only an admiration. Since humans lost their pelage and exposed their skin to sunlight they felt the need to protect themselves, either wearing appropriated clothes or by using paints or powders. As far as we know, the ancients Egyptians were the first to use a kind of sunscreen made by olive oil, and this was also the case in Greece and Rome. However, it was at the end of the nineteenth century when it was reported the first scientific research of a sunscreen protection. From 1887 to 1934 several compounds that can be used as filters of the ultraviolet radiation (UV-filters) were discovered and in 1928 the first sunscreen was commercially available. It was during these years when international cosmetic companies were born (e.g. L'Oreal, Delial, Piz Buin, Coppertone, etc.) and when sunscreens became widely used among population (Rik Roelandts, 2008; Urbach, 2001). After the World War II, a tanned skin became synonymous of good health and beauty.

Nowadays, sunscreens have been shown to give the most effective protection against ultraviolet (UV) radiation damage (Diffey, 2005). A white pale skin has become to be stylish again because people are concerned about skin damage caused by excessive sunlight exposure and thus they have become to use sunscreens all around the globe (Urbach, 2001). Short exposure (between 5 and 10 minutes) to UV radiation has therapeutic effects, i.e. it improves the production of vitamin D and this will increase the calcium absorption, therefore it will prevent from rickets in children and osteoporosis in adults. However, longer exposures can cause severe harm to skin such as skin aging, the erythema (sunburn) and/or melanoma (Holick, 2004).

Three segments compose the sun care market: sun protection, after sun and selftanning. This market increase every year worldwide due to the awareness of the risk associated with the sun exposure ("What's Hot around the Globe: Insights on Personal Care Products. Global Service Studies Website;", 2007). Cosmetic companies flood the market every year with new products, formulations and terms like "nanoparticles", "waterproof" or "broad spectrum" that generates confusion among the user, that are claiming for an effective sunscreen (Kamerow, 2014).

Coastal and marine tourism is the fastest growing sector of the global tourism industry. The growth of this sector includes the development of the infrastructures 
(hotels, ports, second homes, marinas, etc.), water activities and sports (i.e. recreational fishing, yachting, diving, etc.) necessary to accommodate and entertain the large number of visitors to the coastal areas. The impact caused by these activities in the coastal environment has been reported in several studies (Davenport and Davenport, 2006; Gormsen, 1997; Hall, 2001). Thus, environmental degradation and pollution (e.g. by yachts fuel, littering, temporally increase of terrestrial runoff, etc.), destruction of habitats (e.g. destruction of coral reefs due to excessive visitations, disturbance of nearshore aquatic life, etc.) or damage to sand-dune ecosystems (e.g. lost of sandy beaches due to onshore construction) are some of the impacts of coastal tourism.

Despite that coastal tourism is the fastest growing sector in the world (with the consequent increasing in the use of sunscreen), the implications of sunscreens as a source of new chemicals into the coastal marine system have been poorly evaluated.

5

\subsection{Main components of sunscreens}

Sunscreen cosmetic could be defined as: "any cosmetic product containing UVfilters in its formulation in order to protect the skin from the solar deleterious UV-light, avoiding or minimizing the damage that this radiation might cause on human health" (Salvador and Chisvert, 2005). Therefore, the most important components of the sunscreens are the UV-filters: substances with range of light absorption in the range of UVA (400 - $320 \mathrm{~nm})$ and/or UVB (320 - $280 \mathrm{~nm})$ and with nearly null absorption of visible radiation (Díaz-Cruz and Barceló, 2009).

Concentration limits that can be used in sunscreen formulations depend on the different regulations worldwide (Table 1). UV-filters can be organic (classified into different families: i.e. benzophenone derivatives, salicylates, cinnamates, camphor derivatives, p-aminobenzoic acid and its derivatives, etc. (Chisvert and Salvador, 2007)), or inorganic (with only two allowed compounds: titanium dioxide $\left(\mathrm{TiO}_{2}\right)$ and zinc oxide $(\mathrm{ZnO}))$. General usage of these inorganic components in the formulation of sunscreens is in the form of nanoparticles (nano- $\mathrm{TiO}_{2}$ and nano- $\mathrm{ZnO}$, with size around $\leq 100$ nanometers) because they give an effective protection and they do not whiten the skin (Osterwalder et al., 2014). Because its photocatalytic properties, $\mathrm{TiO}_{2}$ nanoparticles used in sunscreens are coated with aluminum oxide or silica to prevent the formation of 
reactive oxygen species (ROS) (Jansen et al., 2013a). Commonly, ZnO in sunscreens are in the form of nanorods while $\mathrm{TiO}_{2}$ are nanoparticles in the rutile structure (Lewicka et al., 2011). L'Oreal Group was the first company that in August 1993 patented the use of nanoparticles of metal oxides as ingredients in sunscreens (Forestier et al., 1995). The action mode of both types of UV-filters are different: while the organic absorbs a specific wavelength, the inorganic ones can give a boarder spectrum protection due to their triple action mode: reflection, scattering and absorption of the UV radiation (Manaia et al., 2013). Normal sunscreens are formed by one or more of these UVfilters: organic, inorganic or a mixture of both. This combination increases the protection giving broad-spectrum sunscreens.

Emollients and emulsifiers are present in an elevated percentage in the composition of sunscreens. Emollients play a triple role in the sunscreens composition: they enable solubilization of some UV-filters (i.e. benzoate esters), photostabilization of unstable UV-filters (i.e. butyloctyl salicylates) and they enhance sensorial feeling in terms of spreading, greasiness, stickiness, etc. (i.e. dicaprylyl carbonate) (Osterwalder et al., 2014). Some emollients can also have an inherent UV absorption that increase the broad spectrum of protection. Emulsifiers are amphiphilic molecules that reduce interfacial tension between two immiscible liquids playing an important role in the stability of the emulsion, consistency, skin feel and care properties of the formulation (Al-Bawab and Friberg, 2006; Plass et al., 2001). The emulsifier system defines the emulsion type: traditionally, oil-in-water $(\mathrm{O} / \mathrm{W})$ or water-in-oil (W/O) system (Osterwalder et al., 2014), besides new formulations of sunscreens became popular among consumers: oils, water-based and hydroalcoholic lotions and microemulsions, also called easy-to-use sunscreens (Chisvert and Salvador, 2007). Anionic emulsifiers such as alkyl phosphates (i.e. potassium cetyl phosphate) are commonly used to stabilize $\mathrm{O} / \mathrm{W}$ emulsions and allow the incorporation of inorganic UV-filters (Miller et al., 1999; Osterwalder et al., 2014). Other emulsifiers such as PEG-30 dipolyhydroxysterate, stabilized W/O systems improving the water resistance of the sunscreens and they may incorporate lipophilic-coated inorganic UV-filters.

In sunscreen composition we can find many other ingredients such as rheology modifiers (thickeners as glycerin or fatty acids), film former agents as acrylates copolymer or silicones, sensory enhancers that improve the skin feeling (i.e. silica, nylon-based compounds, etc.) and in some cases antioxidants such as vitamin $\mathrm{C}$ and 
97 vitamin $\mathrm{E}$, which are included to reduce the oxidative stress generated by ROS formation via UV radiation. Moreover, other photoprotective agents in sunscreens provide protection from erythema and also reduce inflammation and oxidative damage; as for example carotenoids, polyphenols, algae extracts, nicotiamide (amide form of vitamin $\mathrm{B}_{3}$ ), vitamin $\mathrm{A}$ (incorporated as retinyl palmitate), selenium (in the forms of selenium sulfide or L-selenomethionine, that increase the minimal erythema dose), etc.

103 (Chen et al., 2012; Chen and Wang, 2012; Jansen et al., 2013b; Osterwalder et al., 104 2014).

Table 1. Permitted UV-filters according to the different regulations (Source: "Clinical Guide to Sunscreens and Photoprotection”, edited by Henry W. Lim and Zoe Diana Draelos; Informa Healthcare USA, Inc.).

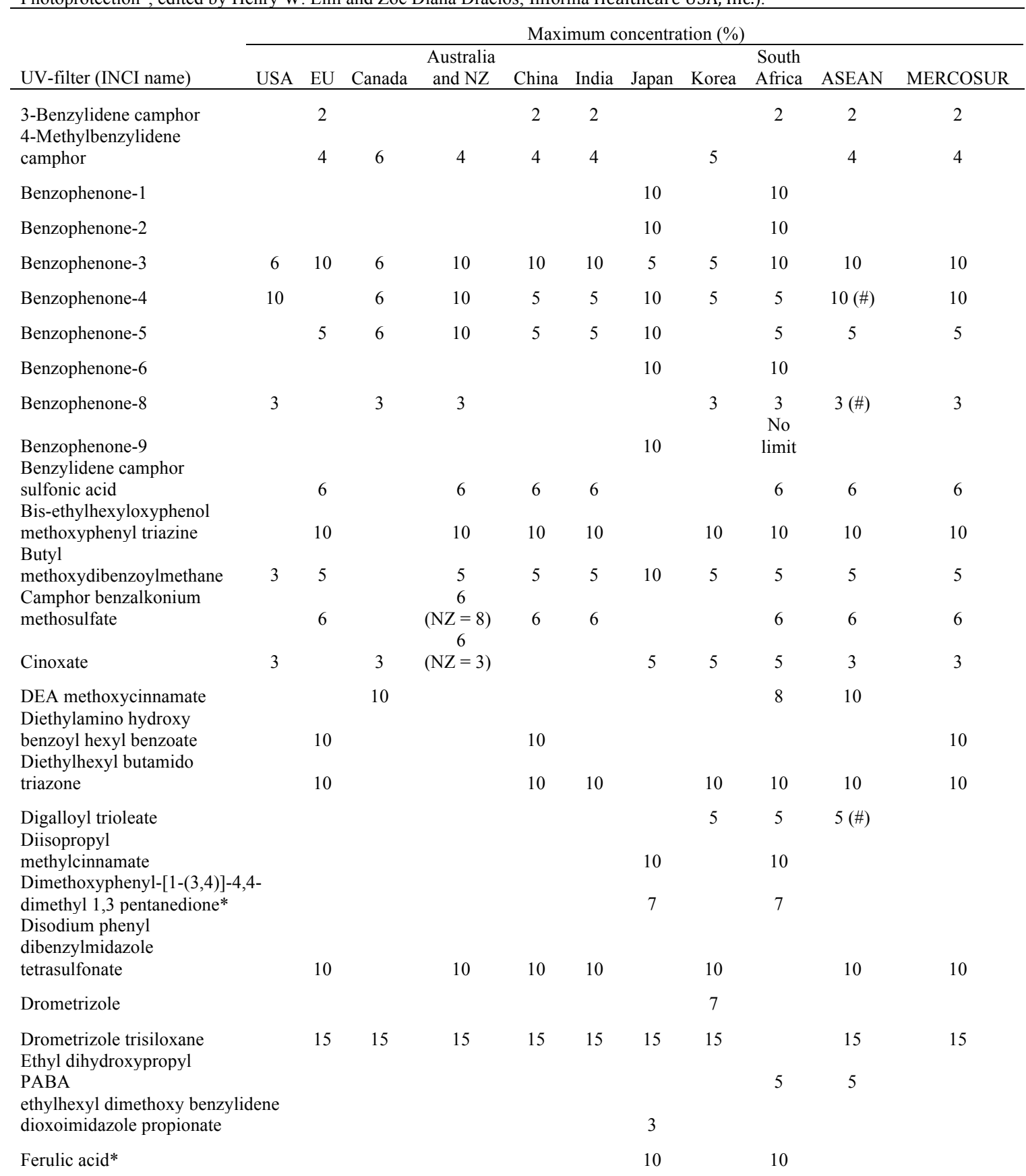




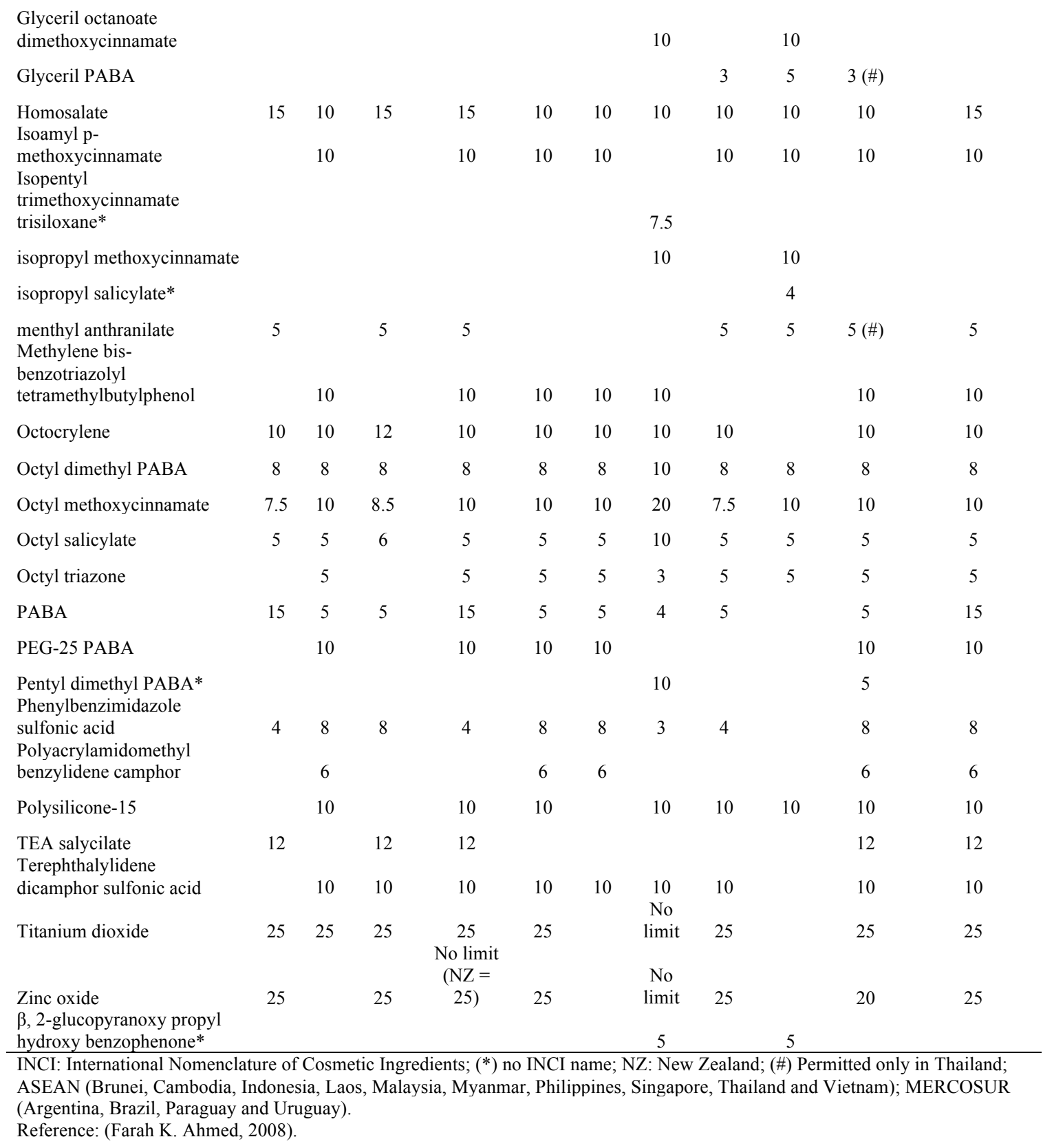
samples

Although the development of sensitive analytical methodologies during last years has allowed the determination of UV-filters and its derivatives in different

110 environmental compartments, information about the presence and concentrations of 111 these components in the marine environment is very scarce.

\subsection{Organic UV-filters}


114 UV-filters in sunscreen composition (Table 1). However, only 16 have been analyzed in 115 environmental matrices (Table 2), therefore the environmental implications of organic 116 UV-filters cannot be completely estimated.

Table 2. Organic UV-filters studied in environmental matrices.

\begin{tabular}{|c|c|c|c|}
\hline & INCI name $^{\mathrm{a}}$ & $\mathrm{CAS} \mathrm{N}^{\mathrm{o}}$ & Empirical formula \\
\hline $3-\mathrm{BC}$ & 3-Benzylidene camphor & $15087-24-8$ & $\mathrm{C}_{17} \mathrm{H}_{20} \mathrm{O}$ \\
\hline 4-MBC & 4-Methylbenzylidene camphor & $36861-47-9$ & $\mathrm{C}_{18} \mathrm{H}_{22} \mathrm{O}$ \\
\hline $\mathrm{BDM}$ & Butyl methoxydibenzoylmethane & 70356-09-1 & $\mathrm{C}_{20} \mathrm{H}_{22} \mathrm{O}_{3}$ \\
\hline BZ-1 & Benzophenone-1 & $131-56-6$ & $\mathrm{C}_{13} \mathrm{H}_{10} \mathrm{O}_{3}$ \\
\hline $\mathrm{BZ}-2$ & Benzophenone-2 & $131-55-5$ & $\mathrm{C}_{13} \mathrm{H}_{10} \mathrm{O}_{5}$ \\
\hline BZ-3 & Benzophenone-3 & $131-57-7$ & $\mathrm{C}_{14} \mathrm{H}_{12} \mathrm{O}_{3}$ \\
\hline BZ-4 & Benzophenone-4 & $4065-45-6$ & $\mathrm{C}_{14} \mathrm{H}_{12} \mathrm{O}_{6} \mathrm{~S}$ \\
\hline BZ-8 & Benzophenone- 8 & $131-53-3$ & $\mathrm{C}_{14} \mathrm{H}_{12} \mathrm{O}_{4}$ \\
\hline HS & Homosalate & $118-56-9$ & $\mathrm{C}_{16} \mathrm{H}_{22} \mathrm{O}_{3}$ \\
\hline IMC & isoamyl p-methoxycinnamate & $71617-10-2$ & $\mathrm{C}_{15} \mathrm{H}_{20} \mathrm{O}_{3}$ \\
\hline OCR & Octocrylene & $6197-30-4$ & $\mathrm{C}_{24} \mathrm{H}_{27} \mathrm{NO}_{2}$ \\
\hline ODP & Octyl dimethyl PABA & $21245-02-3$ & $\mathrm{C}_{17} \mathrm{H}_{27} \mathrm{NO}_{2}$ \\
\hline $\mathrm{OMC}$ & Octyl methoxycinnamate & $5466-77-3$ & $\mathrm{C}_{18} \mathrm{H}_{26} \mathrm{O}_{3}$ \\
\hline OS & Octyl salicylate & $118-60-5$ & $\mathrm{C}_{15} \mathrm{H}_{22} \mathrm{O}_{3}$ \\
\hline OT & Octyl triazone & $88122-99-0$ & $\mathrm{C}_{48} \mathrm{H}_{66} \mathrm{~N}_{6} \mathrm{O}_{6}$ \\
\hline PBS & Phenylbenzimidazole sulphonic acid & $27503-81-7$ & $\mathrm{C}_{13} \mathrm{H}_{10} \mathrm{~N}_{2} \mathrm{O}_{3} \mathrm{~S}$ \\
\hline
\end{tabular}

a INCI (International Nomenclature for Cosmetic Ingredients) elaborated by the Personal Care Products Council (CTFA) and the European cosmetic, toiletry and perfumery industry (COLIPA). CAS $\mathrm{N}^{\mathrm{o}}$ (numerical identifier assigned by Chemical Abstracts Service).

Many analytical methodologies have been developed for the determination of 119 organic UV-filters in different natural matrices (Díaz-Cruz and Barceló, 2009; Salvador 120 and Chisvert, 2005). Due to the very low concentration of organic UV-filters in the 121 environment (ranging from $\mathrm{pM}$ to $\mathrm{nM}$ ), a pre-concentration step is necessary previous to 122 the analysis (mostly based on extraction and microextraction procedures such as 
pressurized liquid extraction, ionic liquid-based single drop microextraction, stir bar

124 sorptive extraction, dispersive liquid-liquid microextraction, solid-phase extraction and 125 microextraction, micelle mediated extraction-solvent back extraction, non-porous 126 membrane-assisted liquid-liquid extraction, etc.). Pre-concentrated analytes are usually 127 separated and quantified by chromatographic techniques coupled with mass 128 spectrometry detection (LC-MS/MS, GC-MS/MS, UHPLC-MS/MS, etc.) (Benedé et 129 al., 2014a, 2014b; Bratkovics and Sapozhnikova, 2011; Cuderman and Heath, 2007; 130 Gago-Ferrero et al., 2013b, 2013c, 2011a; Giokas et al., 2005, 2004; Gómez et al., 131 2009; Lambropoulou et al., 2002; Magi et al., 2012; Nguyen et al., 2011; Oliveira et al., 132 2010; Pedrouzo et al., 2010, 2009; Rodil et al., 2009a, 2009b, 2009c; Rodil and Moeder, 133 2008; Sánchez-Brunete et al., 2011; Tarazona et al., 2010; Trenholm et al., 2008; Vidal 134 et al., 2010; Wick et al., 2010). Passive sampling devices are sometimes used to 135 sampling during large periods of time. For example, the use of semipermeable 136 membrane devices (SPMDs) were used in Swiss lakes and rivers during 3 weeks 137 (Balmer et al., 2005; Buser et al., 2005) and during a cruiser across the Pacific Ocean 138 (Goksøyr et al., 2009).

\subsection{Inorganic UV-filters}

Some analytical approaches have also been proposed for the determination of titanium dioxide nanoparticles as the main inorganic UV-filters in the marine environment. Analytical methodologies for the analysis of nanoparticles in environmental matrices imply separation methods (e.g. size-exclusion chromatography,

144 hydrodynamic chromatography, counter-current chromatography, electrophoresis and 145 capillary electrophoresis or field-flow fractionation), electron microscopy techniques 146 (e.g. transmission electron microscopy, scanning electron microscopy, scanning tunnel 147 microscopy and atomic force microscopy), scattering (dynamic light scattering) and 148 spectroscopy techniques (nuclear magnetic resonance and X-ray spectroscopy) (Farré 149 and Barceló, 2012; López-Heras et al., 2014). In addition to the composition, these 150 techniques provide information about concentration, size distribution, crystallographic structure and morphology. Because of the lack of accuracy methodologies for the analysis of nanomaterials in the complex matrix of seawater, concentration of total $\mathrm{Ti}^{4+}$ after acid digestion is often reported (Luo et al., 2014). In that sense, recently it has been reported a new procedure for the determination of $\mathrm{Ti}^{4+}$ in environmental samples (Sánchez-Quiles et al., 2013). 

lack of reliable methods for its detection and quantification. In addition to the electronic microscopy and particle size techniques (such as hydrodynamic chromatography or electrophoresis), news methods based on asymmetrical flow field-flow fractionation coupled to ICP-MS (asFlFFF-ICP-MS) and single particle ICP-MS (SP-ICP-MS) have been reported for analysis of nano- $\mathrm{TiO}_{2}$ in different environmental samples (Laborda et al., 2014; Lee et al., 2014; López-Heras et al., 2014). Although these methods have demonstrated to be useful for analysis of nanoparticles they have not been tested in seawater samples.

\section{Sunscreens in the environment}

In parallel with the development of new analytical methodologies, ecotoxicological assays have been performed in aquatic organisms in order to establish the toxicity thresholds for these compounds.

It is expected that, on the skin, UV-filters should be photostable under sunlight, however in aqueous media can undergo to a undesirable products that could compromise their UV absorption properties (Santos et al., 2012). Organic UV-filters can undergo to degradation products by different mechanisms: photolysis and photoisomerization, break down by products in wastewater treatment plants (WWTP) or can be metabolized by kidneys after topical application and their metabolites can be excreted in the urine (Díaz-Cruz et al., 2008). UV-filters can reach the marine environment mainly by two different ways: directly as consequence of water recreational activities and indirectly from WWTP effluents (Díaz-Cruz and Barceló, 2009; Giokas et al., 2007).

As a consequence of the increasing coastal tourism and the use of sunscreens the interest of researchers in the determination of organic UV-filters in different environmental and urban compartments has increased in last years (Figure 1). Because

183 WWTP cannot remove efficiently high concentrations of the organic UV-filters, and in spite of the natural degradation that occurs in the environment, these compounds are present in effluents and freshwaters that finally can reach to the sea. Sunscreen organic components have been determined in seawater, freshwaters (rivers and lakes), 
sediments, WWTP influents and effluents, swimming pools, urban groundwater and even in tap water (Table 3). These ingredients even have been found in the lipid tissue of natural populations of aquatic organisms such as mussels, crustacean, eels, fishes, marine mammals and aquatic birds (Table 3), suggesting that bioaccumulation of organic UV-filters in the food webs may be happening.

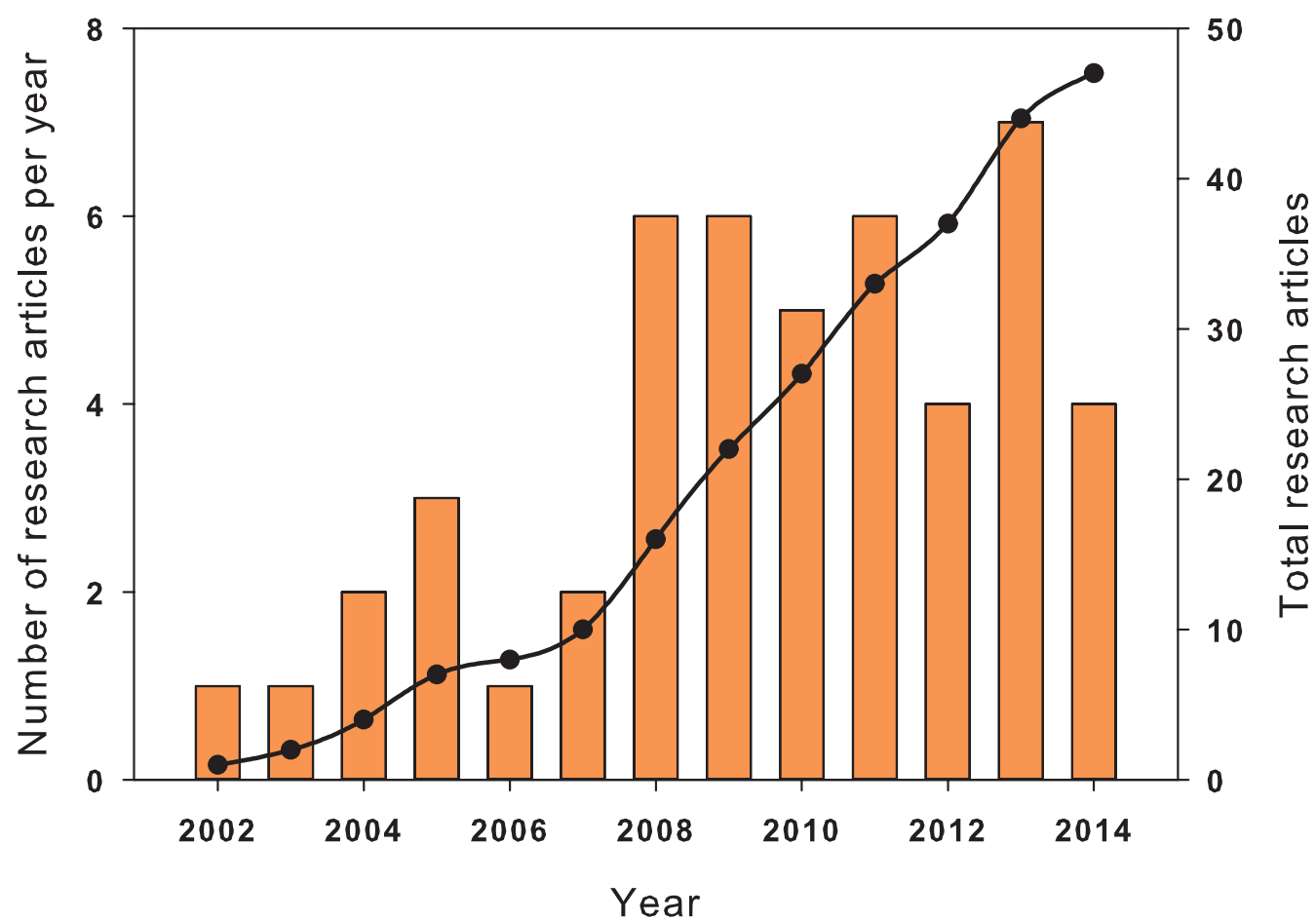

193 Figure 1: Published research articles reporting environmental concentrations of organic 194 UV-filters. Bars represent research articles each year and black line represents the total 195 accumulated articles. 
Table 3. Sample, analytical methodologies, detection limits and range of concentrations of the organic UV-filters in environmental samples.

\begin{tabular}{|c|c|c|}
\hline $\begin{array}{l}\text { Organic } \\
\text { UV-filter }\end{array}$ & Sample & Concentration \\
\hline $3-\mathrm{BC}$ & Seawater* & $9-13 \mathrm{ng} \mathrm{L}^{-1}$ \\
\hline \multirow[t]{20}{*}{ 4-MBC } & Seawater* & n.d. - $798.7 \mathrm{ng} \mathrm{L}^{-1}$ \\
\hline & River water & n.d. - $140 \mathrm{ng} \mathrm{L}^{-1}$ \\
\hline & Lake water & n.d. $-1,140 \pm 50 \mathrm{ng} \mathrm{L}^{-1}$ \\
\hline & WWTP (Influent) & n.d. $-2.7 \mu \mathrm{g} \mathrm{L}^{-1}$ \\
\hline & WWTP (Effluent) & n.d. $-6.5 \mu \mathrm{g} \mathrm{L}^{-1}$ \\
\hline & Swimming pool & n.d. $-330 \mathrm{ng} \mathrm{L}^{-1}$ \\
\hline & Tap water & n.d. - $18 \mathrm{ng} \mathrm{L}^{-1}$ \\
\hline & Urban groundwater & n.d. $-13.9 \mathrm{ng} \mathrm{L}{ }^{-1}$ \\
\hline & Marine sediments & n.d. $-7.90 \mathrm{ng} \mathrm{g}^{-1}$ d.w. \\
\hline & River sediments & n.d. $-17.2 \mathrm{ng} \mathrm{g}^{-1}$ d.w. \\
\hline & Lake sediments & n.d. \\
\hline & STP effluent sediments & n.d. \\
\hline & Beach sand & n.d. $-2.0 \pm 0.4 \mathrm{ng} \mathrm{g}^{-1}$ d.w. \\
\hline & Sewage sludge & $150-4,980 \mathrm{ng} \mathrm{g}^{-1} \mathrm{~d} . \mathrm{w}$. \\
\hline & Coregonus sp. & n.d. \\
\hline & Rutilus rutilus & $44-94$ ng g ${ }^{-1}$ lipid \\
\hline & Perca fluviatilis & 166 ng g $^{-1}$ lipid \\
\hline & Luciobarbus sclareti & n.d. \\
\hline & Cyprinus carpio & n.d. \\
\hline & Leuciscus cephalus/Barbus barbus & n.d. \\
\hline \multirow[t]{6}{*}{$\mathrm{BDM}$} & Seawater & n.d. $-321 \mathrm{ng} \mathrm{L}^{-1}$ \\
\hline & Lake water & $<20-2,431 \pm 22 \mathrm{ng} \mathrm{L}^{-1}$ \\
\hline & WWTP (Influent) & $35.0-1,290.2 \mathrm{ng} \mathrm{L}^{-1}$ \\
\hline & WWTP (Effluent) & n.d. $-1,018.3 \mathrm{ng} \mathrm{L}^{-1}$ \\
\hline & Swimming pool & n.d. \\
\hline & Sewage sludge & $517 \pm 78$ ng g $^{-1}$ d.w. \\
\hline \multirow[t]{13}{*}{ BZ-1 } & Seawater & n.d. $-280 \pm 30 \mathrm{ng} \mathrm{L}^{-1}$ \\
\hline & River water & $<0.3-17 \mathrm{ng} \mathrm{L}^{-1}$ \\
\hline & WWTP (Influent) & n.d. - 722 ng L $^{-1}$ \\
\hline & WWTP (Effluent) & n.d. $-155.0 \mathrm{ng} \mathrm{L}{ }^{-1}$ \\
\hline & Urban groundwater & n.d. $-19.4 n g \mathrm{~L}^{-1}$ \\
\hline & Marine sediments & n.d. \\
\hline & River sediments & n.d. \\
\hline & Agricultural soil & n.d. \\
\hline & Industrial soil & n.d. $-5.7 \pm 0.3 \mathrm{ng} \mathrm{g}^{-1}$ \\
\hline & Activated sludges & $5.1 \pm 1.5 \mathrm{ng} \mathrm{gTSS}^{-1}$ \\
\hline & Sewage sludge & n.d. \\
\hline & Luciobarbus sclareti & n.d. \\
\hline & Cyprinus carpio & n.d. \\
\hline \multirow[t]{2}{*}{ BZ-2 } & River water & n.d. -284 ng L ${ }^{-1}$ \\
\hline & WWTP (Influent) & n.d. $-93 \pm 10 \mathrm{ng} \mathrm{L}^{-1}$ \\
\hline
\end{tabular}




\begin{tabular}{|c|c|c|}
\hline & $\begin{array}{l}\text { WWTP (Effluent) } \\
\text { Urban groundwater } \\
\text { Activated sludges }\end{array}$ & $\begin{array}{c}\text { n.d. - } 14 \pm 3 \text { ng L }^{-1} \\
\text { n.d. } \\
11 \pm 2 \text { ng gTSS }^{-1}\end{array}$ \\
\hline \multirow[t]{29}{*}{ BZ-3 } & Seawater* & n.d. $-3,300 \pm 200 \mathrm{ng} \mathrm{L}^{-1}$ \\
\hline & River water & n.d. -114 ng L $^{-1}$ \\
\hline & Lake water & $<2-125 \mathrm{ng} \mathrm{L}^{-1}$ \\
\hline & WWTP (Influent) & n.d. $-7.8 \mu \mathrm{g} \mathrm{L}^{-1}$ \\
\hline & WWTP (Effluent) & $<0.01-0.7 \mu \mathrm{g} \mathrm{L}^{-1}$ \\
\hline & Swimming pool & n.d. $-3.3 \mu \mathrm{g} \mathrm{L}^{-1}$ \\
\hline & Tap water & n.d. \\
\hline & Urban groundwater & n.d. - $34 \mathrm{ng} \mathrm{L}{ }^{-1}$ \\
\hline & Marine sediments & n.d. $-2.96 \mathrm{ng} \mathrm{g}^{-1}$ d.w. \\
\hline & River sediments & n.d. $-47 \pm 13.1 \mathrm{ng} \mathrm{g}^{-1}$ d.w. \\
\hline & Lake sediments & n.d. \\
\hline & Beach sand & n.d. $-1.0 \pm 0.1 \mathrm{ng} \mathrm{g}^{-1}$ d.w. \\
\hline & Agricultural soil & n.d. \\
\hline & Industrial soil & n.d. \\
\hline & STP effluent sediments & n.d. \\
\hline & Activated sludges & $132 \pm 23 \mathrm{ng}_{\mathrm{gTSS}^{-1}}$ \\
\hline & Sewage sludge & n.d. $-0.79 \mu \mathrm{g} \mathrm{g}^{-1}$ d.w. \\
\hline & Coregonus sp. & n.d. \\
\hline & Rutilus rutilus & $66-118$ ng g $^{-1}$ lipid \\
\hline & Perca fluviatilis & $123 \mathrm{ng} \mathrm{g}^{-1}$ lipid \\
\hline & Luciobarbus sclareti & n.d. $-24.3 \mathrm{ng} \mathrm{g}^{-1}$ d.w. \\
\hline & Cyprinus carpio & $11.2 \mathrm{ng} \mathrm{g}^{-1} \mathrm{~d} . \mathrm{w}$. \\
\hline & Phalacrocorax sp. & n.d. \\
\hline & Dreissena polymorpha & n.d. \\
\hline & Gammarus sp. & n.d. \\
\hline & Leuciscus cephalus & n.d. \\
\hline & Salmo trutta & n.d. $-151 \mathrm{ng} \mathrm{g}^{-1}$ lipid \\
\hline & Barbus barbus & n.d. \\
\hline & Anguilla anguilla & n.d. \\
\hline \multirow[t]{8}{*}{ BZ-4 } & Seawater & $<1 \mathrm{ng} \mathrm{L}^{-1}$ \\
\hline & River water & $<3-1,980 \pm 130 \mathrm{ng} \mathrm{L}^{-1}$ \\
\hline & WWTP (Influent) & n.d. $-4,858 \pm 1,101 \mathrm{ng} \mathrm{L}^{-1}$ \\
\hline & WWTP (Effluent) & n.d. $-1,947 \pm 34$ ng L ${ }^{-1}$ \\
\hline & Tap water & n.d. $-18 \mathrm{ng} \mathrm{L}^{-1}$ \\
\hline & Urban groundwater & n.d. - $36.6 \mathrm{ng} \mathrm{L}^{-1}$ \\
\hline & Activated sludges & $29 \pm 7 \mathrm{ng} \mathrm{gTSS}^{-1}$ \\
\hline & Leuciscus cephalus/Barbus barbus & n.d. \\
\hline \multirow[t]{4}{*}{ BZ-6 } & Marine sediments & n.d. \\
\hline & River sediments & n.d. $-6.1 \pm 0.3 \mathrm{ng} \mathrm{g}^{-1}$ \\
\hline & Agricultural soil & n.d. $-0.6 \pm 0.4 \mathrm{ng} \mathrm{g}^{-1}$ \\
\hline & Industrial soil & n.d. \\
\hline \multirow[t]{2}{*}{ BZ-8 } & Seawater & n.d. \\
\hline & River water & n.d. \\
\hline
\end{tabular}




\begin{tabular}{|c|c|c|}
\hline & $\begin{array}{l}\text { WWTP (Influent) } \\
\text { WWTP (Effluent) } \\
\text { Urban groundwater } \\
\text { Marine sediments } \\
\text { River sediments } \\
\text { Agricultural soil } \\
\text { Industrial soil }\end{array}$ & $\begin{array}{l}\text { n.d. - } 185 \mathrm{ng} \mathrm{L}^{-1} \\
\text { n.d. - } 83.5 \mathrm{ng} \mathrm{L}^{-1} \\
\text { n.d. } \\
\text { n.d. } \\
\text { n.d. } \\
\text { n.d. } \\
\text { n.d. }\end{array}$ \\
\hline HS & $\begin{array}{c}\text { Seawater } \\
\text { River water } \\
\text { Lake water } \\
\text { WWTP (Influent) } \\
\text { WWTP (Effluent) } \\
\text { Swimming pool } \\
\text { Marine sediments } \\
\text { River sediments } \\
\text { Lake sediments } \\
\text { Beach sand } \\
\text { Agricultural soil } \\
\text { Industrial soil } \\
\text { STP effluent } \\
\text { Sewage sludge }\end{array}$ & $\begin{array}{c}\text { n.d. - } 310 \pm 20 \mathrm{ng} \mathrm{L}^{-1} \\
\text { n.d. }-345 \mathrm{ng} \mathrm{L}^{-1} \\
\text { n.d. } \\
\text { n.d. }-1,650.4 \mathrm{ng} \mathrm{L}^{-1} \\
\text { n.d. }-154.2 \mathrm{ng} \mathrm{L}^{-1} \\
\text { n.d. } \\
\text { n.d. } \\
\text { n.d. - } 26 \mu \text { g kg }{ }^{-1} \text { d.w. } \\
\text { n.d. } \\
\text { n.d. }-4.9 \pm 0.7 \mathrm{ng} \mathrm{g}^{-1} \text { d.w. } \\
\text { n.d. } \\
\text { n.d. } \\
\text { n.d. } \\
331 \pm 47 \text { ng g } \\
\end{array}$ \\
\hline IMC & $\begin{array}{c}\text { Seawater } \\
\text { River water } \\
\text { Lake water } \\
\text { WWTP (Influent) } \\
\text { WWTP (Effluent) } \\
\text { Swimming pool } \\
\text { Tap water } \\
\text { Lake sediments } \\
\text { Beach sand } \\
\text { Sewage sludge }\end{array}$ & $\begin{array}{c}\text { n.d. - } 280 \pm 20 \mathrm{ng} \mathrm{L}^{-1} \\
\text { n.d. } \\
146 \pm 20 \mathrm{ng} \mathrm{L}^{-1} \\
\text { n.d. }-226.0 \mathrm{ng} \mathrm{L}^{-1} \\
\text { n.d. }-165.5 \mathrm{ng} \mathrm{L}^{-1} \\
700 \pm 300 \mathrm{ng} \mathrm{L}^{-1} \\
\text { n.d. } \\
\text { n.d. } \\
\text { n.d. }-1.3 \pm 0.3 \mathrm{ng} \mathrm{g}^{-1} \mathrm{~d} . \mathrm{w} . \\
20 \pm 3 \mathrm{ng} \mathrm{g}^{-1} \mathrm{~d} . \mathrm{w} .\end{array}$ \\
\hline OCR & $\begin{array}{c}\text { Seawater } \\
\text { River water } \\
\text { Lake water } \\
\text { WWTP (Influent) } \\
\text { WWTP (Effluent) } \\
\text { Run off water } \\
\text { Swimming pool } \\
\text { Tap water } \\
\text { River sediments } \\
\text { Lake sediments } \\
\text { STP effluent sediments } \\
\text { Sediments } \\
\text { Beach sand } \\
\text { Sewage sludge } \\
\text { Coregonus } s p .\end{array}$ & 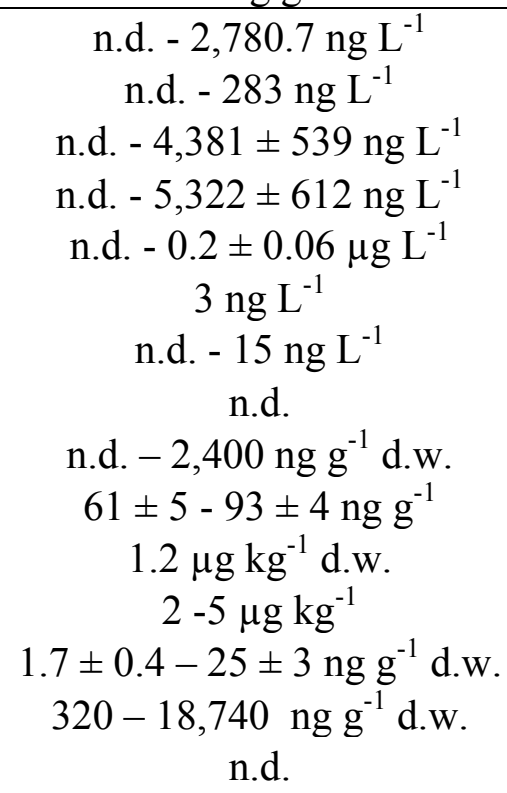 \\
\hline
\end{tabular}


Rutilus rutilus

Perca fluviatilis

Pontoporia blainvillei

Mytilus Sp.

Luciobarbus sclareti

Cyprinus carpio

\begin{tabular}{|c|c|c|}
\hline & Cyprinus carpio & n.d. \\
\hline \multirow[t]{15}{*}{ ODP } & Seawater & n.d. $-390 \pm 40 \mathrm{ng} \mathrm{L}^{-1}$ \\
\hline & River water & n.d. $-47 \mathrm{ng} \mathrm{L}^{-1}$ \\
\hline & Lake water & n.d. $-34 \mathrm{ng} \mathrm{L}^{-1}$ \\
\hline & WWTP (Influent) & n.d. $-376.9 \mathrm{ng} \mathrm{L}{ }^{-1}$ \\
\hline & WWTP (Effluent) & n.d. $-224.3 \mathrm{ng} \mathrm{L}^{-1}$ \\
\hline & Swimming pool & n.d. $-2.1 \mu \mathrm{g} \mathrm{L}^{-1}$ \\
\hline & Tap water & n.d. $-2.3 \mathrm{ng} \mathrm{L}^{-1}$ \\
\hline & Urban groundwater & n.d. \\
\hline & River sediments & n.d. $-17 \pm 3$ ng g $^{-1}$ d.w. \\
\hline & Lake sediments & n.d. \\
\hline & STP effluent sediments & n.d. \\
\hline & Beach sand & n.d. \\
\hline & Sewage sludge & $1.9 \pm 0.3 \mathrm{ng} \mathrm{g}^{-1}$ d.w. \\
\hline & Luciobarbus sclareti & n.d. \\
\hline & Cyprinus carpio & n.d. \\
\hline \multirow[t]{25}{*}{$\mathrm{OMC}$} & Seawater* & n.d. $-389.9 \mathrm{ng} \mathrm{L}^{-1}$ \\
\hline & River water & n.d. - $153 \mathrm{ng} \mathrm{L}^{-1}$ \\
\hline & Lake water & n.d. $-3,009 \pm 206 \mathrm{ng} \mathrm{L}^{-1}$ \\
\hline & WWTP (Influent) & n.d. $-1.9 \mu \mathrm{g} \mathrm{L}^{-1}$ \\
\hline & WWTP (Effluent) & n.d. $-505.2 \mathrm{ng} \mathrm{L}^{-1}$ \\
\hline & Run off water & n.d. \\
\hline & Swimming pool & n.d. $-86 \pm 7 \mathrm{ng} \mathrm{L}^{-1}$ \\
\hline & Tap water & n.d. \\
\hline & Marine sediments & n.d. - 17.8 ng g $^{-1}$ d.w. \\
\hline & River sediments & n.d. $-101 \mu \mathrm{g} \mathrm{kg}^{-1}$ d.w. \\
\hline & Lake sediments & $14 \pm 4-34 \pm 6 \mathrm{ng} \mathrm{g}^{-1}$ \\
\hline & STP effluent sediments & $14 \mu \mathrm{g} \mathrm{kg}^{-1}$ d.w. \\
\hline & Sediments & $34-880 \mu \mathrm{g} \mathrm{kg}^{-1}$ \\
\hline & Beach sand & n.d. $-10 \pm 1 \mathrm{ng} \mathrm{g}^{-1}$ d.w. \\
\hline & Sewage sludge & n.d. $-3.35 \mu \mathrm{g} \mathrm{g}^{-1}$ d.w. \\
\hline & Coregonus sp. & n.d. - $72 \mathrm{ng} \mathrm{g}^{-1}$ lipid \\
\hline & Rutilus rutilus & n.d. - $64 \mathrm{ng} \mathrm{g}^{-1}$ lipid \\
\hline & Perca fluviatilis & n.d. \\
\hline & Mytilus Sp. & $3-256 n g g^{-1}$ d.w. \\
\hline & Luciobarbus sclareti & n.d. $-241.7 \mathrm{ng} \mathrm{g}^{-1}$ d.w. \\
\hline & Cyprinus carpio & n.d. \\
\hline & Phalacrocorax sp. & $16-701 \mathrm{ng} \mathrm{g}^{-1}$ lipid \\
\hline & Dreissena polymorpha & $22-150 \mathrm{ng} \mathrm{g}^{-1}$ lipid \\
\hline & Gammarus sp. & $91-133 \mathrm{ng} \mathrm{g}^{-1}$ lipid \\
\hline & Leuciscus cephalus & $23-79$ ng g $^{-1}$ lipid \\
\hline
\end{tabular}

$25 \mathrm{ng} \mathrm{g}^{-1}$ lipid

n.d. - $712 \mathrm{ng} \mathrm{g}^{-1}$ lipid

n.d. - 7,112 $\mathrm{ng} \mathrm{g}^{-1}$ d.w.

n.d. $-30.4 \mathrm{ng} \mathrm{g}^{-1}$ d.w.

n.d.

$-390 \pm 40 \mathrm{ng} \mathrm{L}^{-1}$

n.d. - $47 \mathrm{ng} \mathrm{L}^{-1}$

n.d. - 34 ng $L^{-1}$

n.d. - $376.9 \mathrm{ng} \mathrm{L}^{-1}$

d. $-224.3 \mathrm{ng} \mathrm{L}^{-1}$

n.d. $-2.1 \mu \mathrm{g} \mathrm{L}$

n.d.

$3 \mathrm{ng} \mathrm{g}^{-1} \mathrm{~d}$.w.

$3 \mathrm{ng} \mathrm{g}^{-1}$ d.w.

n.d. $-389.9 \mathrm{ng} \mathrm{L}{ }^{-1}$

$-3,009 \pm 206 \mathrm{ng} \mathrm{L}^{-1}$

n.d. $-1.9 \mu \mathrm{g} \mathrm{L}^{-1}$

$05.2 \mathrm{ng} \mathrm{L}^{-1}$

$6 \pm 7 \mathrm{ng} \mathrm{L}^{-1}$

n.d. $-17.8 \mathrm{ng} \mathrm{g}^{-1}$ d.w.

n.d. $-101 \mu \mathrm{g} \mathrm{kg}^{-1}$ d.w

$\pm 4-34 \pm 6 \mathrm{ng} \mathrm{g}^{-1}$

$14 \mu \mathrm{g} \mathrm{kg}^{-1}$ d.w.

.d. $-10 \pm 1 \mathrm{ng} \mathrm{g}^{-1}$ d.w.

n.d. $-3.35 \mu \mathrm{g} \mathrm{g}^{-1}$ d.w.

n.d. - $72 \mathrm{ng} \mathrm{g}^{-1}$ lipid

ng

3 - $256 \mathrm{ng} \mathrm{g}^{-1}$ d.w.

.

$16-701 \mathrm{ng} \mathrm{g}^{-1}$ lipid

$22-150 \mathrm{ng} \mathrm{g}^{-1}$ lipid

$23-79 \mathrm{ng} \mathrm{g}^{-1}$ lipid 


\begin{tabular}{|c|c|c|}
\hline & $\begin{array}{c}\text { Salmo trutta } \\
\text { Barbus barbus } \\
\text { Anguilla anguilla } \\
\text { Leuciscus cephalus/Barbus barbus }\end{array}$ & $\begin{array}{c}\text { n.d. }-205 \mathrm{ng} \mathrm{g}^{-1} \text { lipid } \\
\text { n.d. }-337 \mathrm{ng} \mathrm{g}^{-1} \text { lipid } \\
30 \mathrm{ng} \mathrm{g}^{-1} \text { lipid } \\
4 \pm 5-142 \pm 95 \mathrm{ng} \mathrm{g}^{-1} \text { lipid }\end{array}$ \\
\hline \multirow[t]{14}{*}{ OS } & Seawater & n.d. $-880 \pm 30 \mathrm{ng} \mathrm{L}^{-1}$ \\
\hline & River water & n.d. -266 ng L ${ }^{-1}$ \\
\hline & Lake water & $748 \pm 60 \mathrm{ng} \mathrm{L}^{-1}$ \\
\hline & WWTP (Influent) & n.d. $-1,188.3 \mathrm{ng} \mathrm{L}^{-1}$ \\
\hline & WWTP (Effluent) & n.d. $-128.9 \mathrm{ng} \mathrm{L}^{-1}$ \\
\hline & Swimming pool & n.d. \\
\hline & Marine sediments & $13.3 \pm 0.4 \mathrm{ng} \mathrm{g}^{-1}$ \\
\hline & River sediments & n.d. $-20.0 \pm 0.5 \mathrm{ng} \mathrm{g}^{-1}$ \\
\hline & Lake sediments & n.d. \\
\hline & Beach sand & $1.8 \pm 0.5-12 \pm 1 \mathrm{ng} \mathrm{g}^{-1}$ d.w. \\
\hline & Agricultural soil & n.d. \\
\hline & Industrial soil & n.d. \\
\hline & STP effluent sediments & n.d. \\
\hline & Sewage sludge & $280 \pm 37 \mathrm{ng} \mathrm{g}^{-1}$ d.w. \\
\hline OT & Sewage sludge & $700-27,700$ ng g $^{-1}$ d.w. \\
\hline \multirow[t]{6}{*}{ PBS } & Seawater & n.d. \\
\hline & River water & $48 \pm 3-3,240 \pm 140 \mathrm{ng} \mathrm{L}^{-1}$ \\
\hline & WWTP (Influent) & $196 \pm 56-3,890 \pm 170 \mathrm{ng} \mathrm{L}^{-1}$ \\
\hline & WWTP (Effluent) & n.d. $-1,820 \pm 240 \mathrm{ng} \mathrm{L}^{-1}$ \\
\hline & Tap water & n.d. \\
\hline & Activated sludges & n.d. \\
\hline
\end{tabular}

* Including surface microlayer; River water (river and stream); n.d. (no detected); d.w. (dry weight); WWTP (waste water treatment plant); STP (sewage treatment plant).

References: (Amine et al., 2012; Arukwe et al., 2012; Bachelot et al., 2012; Balmer et al., 2005; Barón et al., 2013; Benedé et al., 2014b; Bratkovics and Sapozhnikova, 2011; Buser et al., 2005; Cuderman and Heath, 2007; Fent et al., 2010b; Gago-Ferrero et al., 2013a, 2013b, 2013c, 2011a, 2011b; Giokas et al., 2005, 2004; Goksøyr et al., 2009; Gómez et al., 2009; Jurado et al., 2014; Kameda et al., 2011; Kasprzyk-Hordern et al., 2008; Kawaguchi et al., 2008; Lambropoulou et al., 2002; Langford and Thomas, 2008; Li et al., 2007; Magi et al., 2012; Nguyen et al., 2011; Pedrouzo et al., 2010, 2009; Pintado-Herrera et al., 2013; Plagellat et al., 2006; Poiger et al., 2004; Ricking et al., 2003; Rodil et al., 2009a, 2009b, 2009c; Rodil and Moeder, 2008; Sánchez-Brunete et al., 2011; Tarazona et al., 2014, 2010; Tashiro and Kameda, 2013; Tovar-Sánchez et al., 2013; Trenholm et al., 2008; Tsui et al., 2014; Vidal et 203 al., 2010; Wick et al., 2010; Zenker et al., 2008)

Many ecotoxicological studies have been done to assess the potential damage of

205 sunscreens and their components. In vitro experiments have demonstrated that organic

206 UV-filters might be toxic for some aquatic microorganism. For example, Sieratowicz et

207 al. (2011) calculated the half maximal inhibitory concentration $\left(\mathrm{IC}_{50}\right)$ of benzophenone-

208 (BZ-3), 3-bencylidene camphor (3-BC) and 4-methyl bencylidene camphor (4-MBC) 
209 for a freshwater phytoplankton specie Desmodesmus subspicatus $\left(0.96 \mathrm{mg} \mathrm{L}^{-1}, 6.99 \mathrm{mg}\right.$ $210 \mathrm{~L}^{-1}$ and $7.66 \mathrm{mg} \mathrm{L}^{-1}$, respectively) in 72 hours culture experiment. More recently, 211 Paredes et al. (2014) have calculated the toxicity according to the half maximal 212 effective concentration $\left(\mathrm{EC}_{50}\right)$ in the specie Isochyris galbana concluding that toxicity 213 decreases from BZ-3, octyl methoxycinnamate (OMC), 4- MBC and benzophenone-4 214 (BZ-4) with $\mathrm{EC}_{50}$ of $13.87 \mathrm{ng} \mathrm{mL}^{-1}, 74.72 \mathrm{ng} \mathrm{mL}^{-1}, 171,45 \mathrm{ng} \mathrm{mL}^{-1}$ and $>10,000 \mathrm{ng}$ $215 \mathrm{~mL}^{-1}$, respectively. In the protozoan Tetrahymena thermophila BZ-3 and 4-MBC could 216 inhibit the growth ( $\mathrm{EC}_{50}$ of $7.5 \mathrm{mg} \mathrm{L}^{-1}$ and $5.1 \mathrm{mg} \mathrm{L}^{-1}$, respectively) in $24 \mathrm{~h}$ of culture 217 experiments (Gao et al., 2013). The ecotoxicity of UV-filters in crustacean Daphnia 218 magna showed after $48 \mathrm{~h}$ that OMC was more toxic than 4-MBC, BZ-3, 3-BC and BZ-4 $219\left(\mathrm{EC}_{50}\right.$ of $0.29-0.57 \mathrm{mg} \mathrm{L}^{-1}, 0.56-0.80 \mathrm{mg} \mathrm{L}^{-1}, 1.67-1.9 \mathrm{mg} \mathrm{L}^{-1}, 3.61 \mathrm{mg} \mathrm{L}^{-1}$ and 50 $220 \mathrm{mg} \mathrm{L}^{-1}$, respectively) (Fent et al., 2010a; Sieratowicz et al., 2011).

Evaluated the toxicity of four UV-filters in marine invertebrates, Mytilus galloprovincialis (mussels), Paracentrotus lividus (sea urchins) and Siriella armata (crustacea), the following conclusion has been reached: OMC and 4-MBC were the most toxics, whereas BZ-4 presented the lowest toxicity (Paredes et al., 2014). Also OMC showed toxic effects on snails (Melanoides tuberculata and Potamopyrgus antipodarum) while butyl methoxydibenzoylmethane (BDM) and octocrylene (OCR) showed no effects (Kaiser et al., 2012).

Hormonal effects (estrogenic, antiestrogenic, androgenic and antiandrogenic activities) of some organic UV-filters have been extensively studied using in vitro test in human cells, fishes and frogs (Díaz-Cruz and Barceló, 2009; Kim and Choi, 2014 and references therein). Different studies carried out in fishes have demonstrated that concentrations of organic UV-filters may induce change in genes in hormonal pathways, for example concentrations in the order of $\mu \mathrm{g} \mathrm{L}^{-1}$ of BZ-3, BZ-4 or OMC down-regulated the expression of genes involved in the sex hormone of Danio rerio (zebrafish) at two different life stages, eleuthero-embryos and adult (Blüthgen et al., 2012; Zucchi et al., 2011a, 2011b).

Due to its composition, sunscreens are a source of $\mathrm{H}_{2} \mathrm{O}_{2}$ into the coastal marine waters. It has been demonstrated that under UV radiation some organic UV-filters (e.g. BZ-3, OCR, OMC, PBSA, PABA, etc.) can generate ROS $\left(\mathrm{O}_{2}{ }^{-}, \mathrm{OH} \cdot, \mathrm{H}_{2} \mathrm{O}_{2}\right.$, etc.) (Allen 240 et al., 1996; Hanson et al., 2006; Inbaraj et al., 2002). Sánchez-Quiles and Tovar- 
241 Sánchez (2014) demonstrated that sunscreens may increase the concentration of $\mathrm{H}_{2} \mathrm{O}_{2}$ 242 up to $270 \mathrm{nM} /$ day in a Mediterranean Beach. These oxidizing species can damage 243 lipids, proteins and DNA and they can generate high stress levels in marine organisms 244 (Lesser, 2006, and references therein). But not only these organic UV-filters can 245 generate ROS, other studies point out that inorganic UV-filters (i.e. $\mathrm{TiO}_{2}$ and $\mathrm{ZnO}$ ), 246 under UV radiation results toxic for the marine phytoplankton. Many studies agree that 247 the toxicity of nano- $\mathrm{TiO}_{2}$ is produced by its photochemical properties under solar 248 radiation, that depends on the radiation intensity and the crystalline structure and 249 concentration of the nanoparticles (Hund-Rinke and Simon, 2006; Li et al., 2014; 250 Mansfield et al., 2015). Even though nanoparticles are usually covered with an inert 251 coating layer to avoid its photoreactivity, this layer is dissolved in aquatic environments 252 after being released from sunscreens (Botta et al., 2011; Labille et al., 2010). A recently 253 published review about phototoxicity of nano- $\mathrm{TiO}_{2}$ calculates the "phototoxicity ratio" 254 obtained with experiments conducted in presence and absence of sunlight and concludes 255 that phototoxicity of nano- $\mathrm{TiO}_{2}$, under solar radiation, is specially harmful for the order 256 Cladocera (Jovanović, 2015).

Very little is known about the worldwide production of these two oxide 258 nanoparticles. In accordance with the United States Environmental Protection Agency 259 (US EPA), in 2005 the global production of nano- $\mathrm{TiO}_{2}$ was estimated at 2,000 tons and 260 during the period 2006-2010 has been calculated at 5,000 tons per year (US EPA 261 National Center for Environmental Assessment and Powers). However, there are others 262 estimations of this production, thus Piccinno et al. (2012) estimated, based on an 263 industry survey, a global production of 550 to 5,500 tons per year. According to 264 Aschberger et al. (2011) the estimated production is about 60,000 tons per year. The 265 global production of nano-ZnO was calculated also in several studies, thus while 266 Piccinno et al. (2012) calculated a production between 55 and 550 tons per year, 267 Aschberger et al. (2011) estimated in 10,000 tons per year the nano- $\mathrm{ZnO}$ production. It 268 is believed that $60 \%$ of nano- $\mathrm{TiO}_{2}$ and $80 \%$ of nano- $\mathrm{ZnO}$ of the global production are 269 used in cosmetic products (Piccinno et al., 2012). These nanoparticles can reach the 270 marine environment during their entire life cycle (i.e. production of nanoparticles, 271 fabrication and use of products) via air deposition, WWTP effluents and/or direct 272 release (Baker et al., 2014; Sun et al., 2014). Once in the seawater they can interact with 273 aquatic organisms in different ways: adsorption to the surface of microorganisms, 
cellular internalization, trapping by filter feeder organisms (e.g. bivalves), ingest by benthic fauna from the sediments or uptake by fishes (Baker et al., 2014). SánchezQuiles and Tovar-Sánchez (2014) estimated that in a touristic beach during a summer day about $4 \mathrm{~kg}$ of $\mathrm{TiO}_{2}$ nanoparticles could be released from sunscreens into seawater. Other authors suggest that recreational activities that take place at the Old Danube Lake may imply a consumption of sunscreen of 8.1 tons per year, and estimated that $94.5 \mathrm{Kg}$ of $\mathrm{TiO}_{2}$ per year may released in the lake waters (Gondikas et al., 2014).

Many studies provide evidence of the toxicity of nanoparticles in aquatic organisms and most of them (64\%) were performed on fresh waters species, and only $14 \%$ were on salt water species (Minetto et al., 2014). Miller et al. (2010) demonstrated that nano- $\mathrm{ZnO}$ produces inhibition growth in four species of marine phytoplankton with concentrations between 0.5 and $1.0 \mathrm{mg} \mathrm{L}^{-1}$, while nano- $\mathrm{TiO}_{2}$ does not present any significantly effects at these concentrations. Ma et al. (2012) demonstrated that phototoxicity of nano- $\mathrm{TiO}_{2}$ increased between 2 to 4 times under simulating solar radiation, being Daphnia magna 100 times more sensible than fish Japanese medaka (Oryzias latipes). The effects of long-term exposure of cladoceran Daphnia magna to nano- $\mathrm{TiO}_{2}$ were studied over six generations showing that chronic exposure to $1.78 \mathrm{mg}$ $\mathrm{L}^{-1}$ of nano- $\mathrm{TiO}_{2}$ resulted in $100 \%$ mortality (Jacobasch et al., 2014). A similar study in zebrafish (Danio rerio) demonstrated that, after six months of exposure, nano- $\mathrm{TiO}_{2}$ was bioaccumulated in brain, gill, liver and heart, producing organ injury and mortality 294 (Chen et al., 2011).

To our knowledge, few studies address the environmental implications of the totally composition of sunscreens. The first study that provided the toxic effect of sunscreens in the marine environment was carried out by Danovaro and Corinaldesi (2003), who demonstrated that sunscreens induce viral infections and control marine bacterioplankton. The same authors demonstrated that sunscreens affect corals bleachings by promoting the lytic viral cycle, killing the symbiotic microalgae zooxanthellae (Danovaro et al., 2008). On the other hand, Tovar-Sánchez et al. (2013) demonstrated, with laboratory experiments and field measurements, that sunscreens are an important source of nutrients (nitrate $\left(\mathrm{NO}_{3}{ }^{-}\right)$, nitrite $\left(\mathrm{NO}_{2}{ }^{-}\right)$, phosphate $\left(\mathrm{PO}_{4}{ }^{3-}\right)$, silicate $\left(\mathrm{SiO}_{2}\right)$ and ammonium $\left.\left(\mathrm{NH}_{4}^{+}\right)\right)$to the coastal marine environment that could enhance the primary production in the oligotrophic waters of the Mediterranean Sea. 
A study carried out on a touristic beach indicates that exist a temporal (daily) and vertical (water column) distribution of UV chemical filters and $\mathrm{H}_{2} \mathrm{O}_{2}$ concentrations in coastal waters, with the highest concentrations of UV-filters measured in the surface microlayer (SML) (Sánchez-Quiles and Tovar-Sánchez, 2014; Tovar-Sánchez et al., 2013). According to these authors, concentrations of chemicals in the first centimeter of the sea surface water (SML) were up to $41.5 \%$ for BZ-3, $43.0 \%$ for $4-\mathrm{MBC}$ and 41.6 $\%$ for $\mathrm{H}_{2} \mathrm{O}_{2}$ higher than in the immediately subsurface waters. Because of its lipophilicity (e.g. $\log \mathrm{K}_{\mathrm{ow}}$ of 3.79 and 4.95 for BZ-3 and 4-MBC, respectively) these compounds tend to accumulate in the SML and in muscle and adipose tissues of marine organisms (Gago-Ferrero et al., 2012), thus elevated concentrations of OCR were found in the liver of Franciscana dolphin (Pontoporia blainvillei) from Brazil (up to $712 \mathrm{ng} \mathrm{g}^{-1}$ lipid) (Gago-Ferrero et al., 2013a) or in mussels (Mytilus Sp.) collected along the French coast (up to 7,112 $\mathrm{ng} \mathrm{g}^{-1}$ d.w.) (Bachelot et al., 2012) (Table 3).Goksøyr et al. (2009) reported concentrations of organic UV-filters in open waters of the Pacific, providing evidence of the persistence and wide dispersion of these components in the marine environment.

The toxic effects of sunscreens and their main components have opened a debate about the regulation and labeling of sunscreens. In accordance with their chemical composition (Sobek et al., 2013) suggested that sunscreens should be labeled according to the European Union CLP regulation (classification, labeling and packaging; EC 1272/2008). Therefore sunscreens with ingredients that could be a risk for marine environment (e.g. nano- $\mathrm{TiO}_{2}$ ), should be labeled with hazard statements or even labeled with hazard pictograms, as occurs with other products that include the same components, such as paints. On the other hand, the Environmental Working Group (EWG) affirm that sunscreens with inorganic UV-filters results a better choice for marine environment than those with organic UV-filters (EWG, 2014), although they are aware that effects of nanoparticles in the environment remains unknown. Because of the effects of nanoparticles in the marine ecosystem are not fully understood, Jacobs et al. (2010) considered that the use of these nanoparticles in sunscreens are morally unacceptable. Consequently, investigations of sunscreens with "environmental-friendly" formulations have increased in the last years (Danovaro et al., 2014). Since many 337 organisms in nature have developed its own photoprotective mechanisms the use of 338 natural components is being explored. Several types of secondary metabolites are 
known to act as sunscreens in plants and animals, such as melanin (in humans), scytonemin (in cyanobacteria), mycosporine and mycosporine-like amino acids (MAA's) (with an wide phylogenetic distribution) and carotenoids which biosynthesis can be UVB-inducible in cyanobacteria (Gao and Garcia-Pichel, 2011, and references therein).

\section{Coastal Tourism trend and use of sunscreen: the Spain case}

The world coastal-zone population is expected to grow from 1.2 billion people (in 1990) up to 5.2 billion by the 2080 (Rabalais et al., 2009). According to the Word Tourism Organization (WTO), the Mediterranean coast received almost 30\% of world international tourist arrivals in 2013 (UNWTO World Tourism Barometer, 2014). With 220 million of tourists per year the Mediterranean region is one of the most visited destinations in the world, and half of these visitors attend en masse to the Mediterranean beaches (WWF Global). Moreover, it has been estimated that the Mediterranean region could receive up to 264 million tourists in 2030 (World Tourism Organization UNWTO, 2011).

In Spain, tourism is one of the mainstays of the country economy. It means around $11 \%$ of GDP and $13 \%$ of employment, and contributes substantially to offsetting the trade deficit. With a new record of foreign visitors (i.e. 64.9 million in 2014) (Turespaña, http://www.iet.tourspain.es), Spain has been consolidated as the second-largest worldwide destination (in the OECD countries: organization for Economic Co-operation and Development) in terms of tourist arrivals and receipts. Tourist arrivals have increased by 93\% in the period 2004 - 2008 (OECD Tourism Trends and Policies, 2010), with more than $50 \%$ of arrivals concentrated in the Mediterranean coasts (Catalonia, Balearic Islands and Valencia) (Mantiñán and Solla, 2010). In fact, Balearic Islands is one of the most important tourist destinations in the Mediterranean Sea (Cirer-Costa, 2012). The international tourist arrivals are increasing every year, with more than 11 million of tourists arrivals in 2013, mostly concentrated during summer season (Turespaña, http://www.iet.tourspain.es). In fact, it has been considered like a second generation European mass tourist resort (Knowles and Curtis, 1999). With a 1.1 million of inhabitants, this income of visitors counts as more than 10 times the normal population of the islands 
371 fastest growing sales rate globally, with the Western Europe as the largest market. Sun 372 protection is the most important segment of the sun care market with almost $€ 7.0$ 373 billion forecasted in 2014, a worldwide increase of $7 \%$ per year over the last five years, 374 and with an average per capita consumption of $20 \mathrm{ml}$ per year (Osterwalder et al., 375 2014). Despite the high diversity of textures of sunscreens (e.g. lotions, spray, roll-on, 376 shake well types, etc.) lotions and creams represent approximately $60 \%$ of the total 377 products introduced in the market. Spray format is also very popular in Europe with 20 $378 \%$ of presence in the market. Sunscreens with Solar Protection Factor (SPF) between 30 $379-50$ are produced in high quantities, and they represent the $50-60 \%$ (Osterwalder et 380 al., 2014). Spain is the country with the highest consume of sunscreen per capita with $381189 \mathrm{ml}$ per year in 2012 (Osterwalder et al., 2014). Considering 47 million of 382 inhabitants it would represent a consumption of almost 9 million liters of sunscreen per 383 year. According to a survey carried out in Spain, almost $80 \%$ of the population used 384 385 386 sunscreen to protect their skin against the UV radiation (Galán et al., 2011). This percentage is significantly higher than in the United Kingdom (37\%) (Miles et al., 2005 ) or Australia (27\%) (Dobbinson et al., 2008).

Despite this data, little is known about the effects of sun-care products on the marine ecosystems. The use of these cosmetics has become popular since the second half of 20th century; however, the first analyses of the UV-filters environmental samples were made in 2002 (Lambropoulou et al., 2002). With less than 50 peer-review scientific articles published to date (Figure 1), Spain is the country which has the highest number of research investigations involving the analysis of environmental levels of organic UV-filters (Figure 2). 


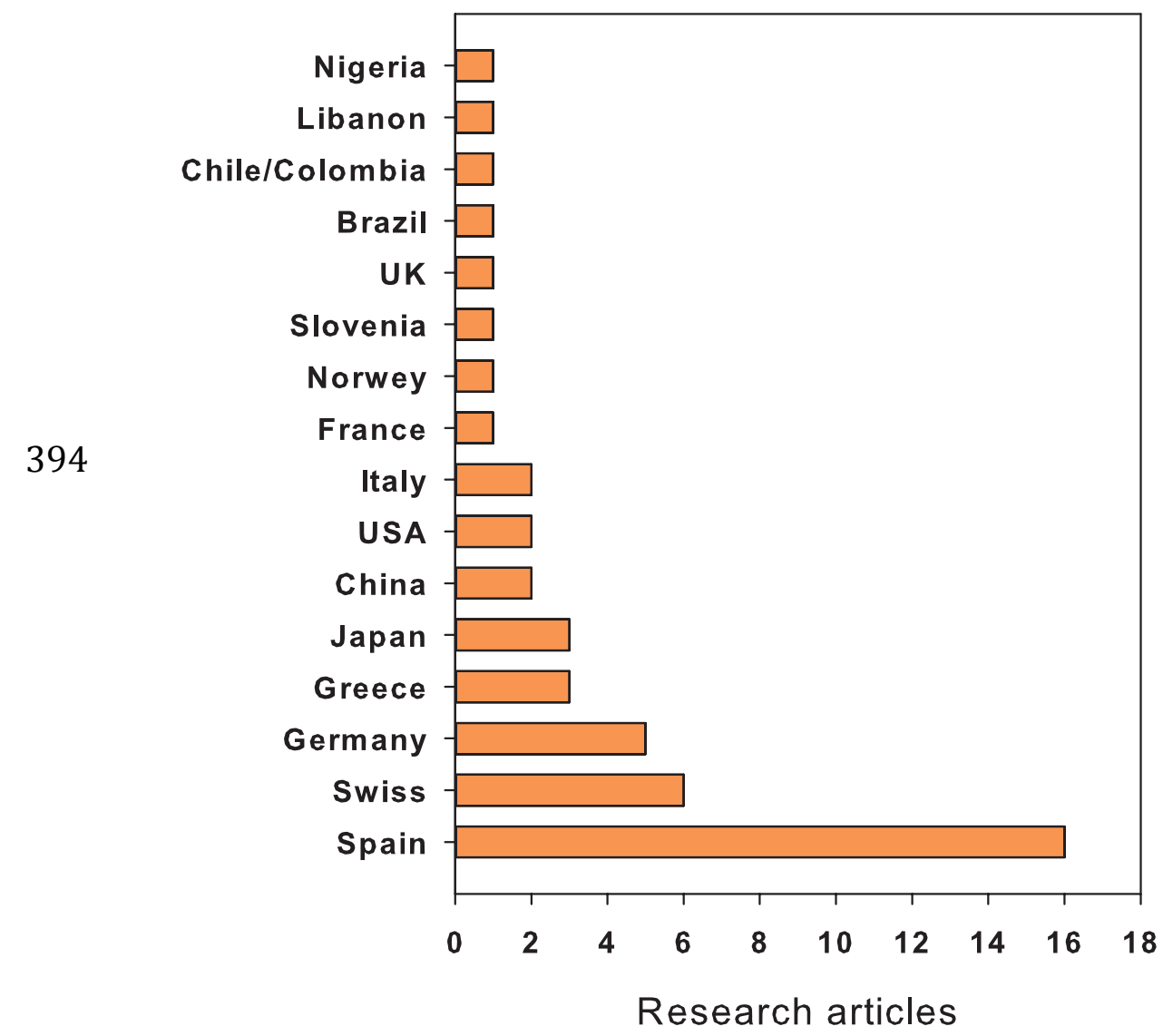

395 Figure 2: Published research articles reporting environmental concentrations of organic 396 UV-filters sorted by country.

\section{5. Conclusions and future perspectives}

This review evaluates the environmental implications of commercial sunscreens and their main components as source of chemicals into the coastal ecosystem. Results presented here indicate that sunscreens are a significant source of chemicals that reach the sea and have potential ecological consequences on the coastal marine ecosystem.

Once in the water column, components released from sunscreens accumulate in the SML. Organic and inorganic UV-filters are photo-excited by sunlight generating elevated concentrations of reactive oxygen species with toxic effects on phytoplankton and being potentially bioaccumulative in the food web. Other components released from sunscreen $\left(\mathrm{NO}_{3}{ }^{-}, \mathrm{NO}_{2}{ }^{-}, \mathrm{PO}_{4}{ }^{3-}, \mathrm{SiO}_{2}\right.$ and $\left.\mathrm{NH}_{4}{ }^{+}\right)$are easily dissolved in seawater and may stimulate algal growth (Figure 3). 
pollutant in the marine system, such as distribution and partitioning in the water column, dissolution and speciation of their main components, evaluation of the ecological significance of the input of nutrients, residence time and aging, persistence, accumulation and toxicity in the trophic chain. Additional ecotoxicological experiments are also needed to better understand the effects of these products in the marine environment. For example, new studies should evaluate the stress level and species succession in marine phytoplankton species and the effect on macroalgae and marine seagrasses. The marine macrophyte Posidonia oceanica, is an endemic seagrass from the Mediterranean Sea that is included in the Annex II of the Protocol of Barcelona Convention 1996 as an endangered marine species. The nearshore habitat of these marine plants, together with many other pelagic and benthic fauna, receives the influence of sunscreens and therefore its impact should be addressed.

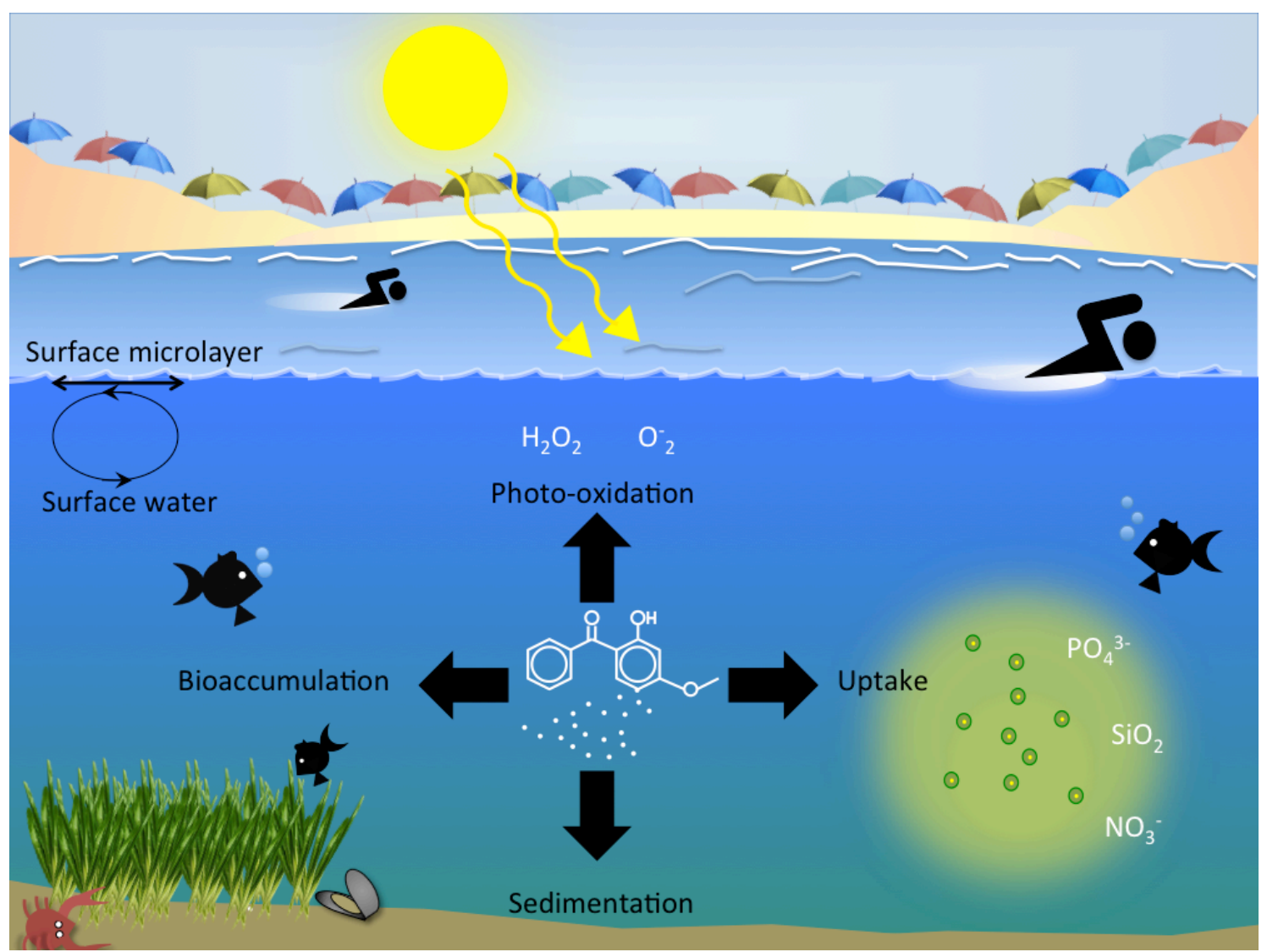

Figure 3: Conceptual diagram transfer of sunscreen derived-products. 
428 Acknowledgements

429 The authors thank Marisu Sánchez for helping with manuscript edition. D.S.-Q. was 430 supported by the JAE-predoc program of the Spanish National Research Council 431 (CSIC). 


\section{References}

Al-Bawab, A., Friberg, S.E., 2006. Some pertinent factors in skin care emulsion. Adv. Colloid Interface Sci., Special Issue in Honor of Dr. K. L. Mittal 123-126, 313-322. doi:10.1016/j.cis.2006.06.004

Allen, J.M., Gossett, C.J., Allen, S.K., 1996. Photochemical formation of singlet molecular oxygen in illuminated aqueous solutions of several commercially available sunscreen active ingredients. Chem. Res. Toxicol. 9, 605-609. doi:10.1021/tx950197m

Amine, H., Gomez, E., Halwani, J., Casellas, C., Fenet, H., 2012. UV filters, ethylhexyl methoxycinnamate, octocrylene and ethylhexyl dimethyl PABA from untreated wastewater in sediment from eastern Mediterranean river transition and coastal zones. Mar. Pollut. Bull. 64, 2435-2442. doi:10.1016/j.marpolbul.2012.07.051

Arukwe, A., Eggen, T., Möder, M., 2012. Solid waste deposits as a significant source of contaminants of emerging concern to the aquatic and terrestrial environments - A developing country case study from Owerri, Nigeria. Sci. Total Environ. 438, 94-102. doi:10.1016/j.scitotenv.2012.08.039

Aschberger, K., Micheletti, C., Sokull-Klüttgen, B., Christensen, F.M., 2011. Analysis of currently available data for characterising the risk of engineered nanomaterials to the environment and human health - Lessons learned from four case studies. Environ. Int., Special Issue: Environmental Fate and Effects of Nanoparticles 37, 1143-1156. doi:10.1016/j.envint.2011.02.005

Bachelot, M., Li, Z., Munaron, D., Le Gall, P., Casellas, C., Fenet, H., Gomez, E., 2012. Organic UV filter concentrations in marine mussels from French coastal regions. Sci. Total Environ. 420, 273-279. doi:10.1016/j.scitotenv.2011.12.051

Baker, T.J., Tyler, C.R., Galloway, T.S., 2014. Impacts of metal and metal oxide nanoparticles on marine organisms. Environ. Pollut. Barking Essex 1987 186C, 257271. doi:10.1016/j.envpol.2013.11.014

Balmer, M.E., Buser, H.-R., Müller, M.D., Poiger, T., 2005. Occurrence of Some Organic UV Filters in Wastewater, in Surface Waters, and in Fish from Swiss Lakes. Env. Sci Technol 39, 953-962. doi:10.1021/es040055r

Barón, E., Gago-Ferrero, P., Gorga, M., Rudolph, I., Mendoza, G., Zapata, A.M., DíazCruz, S., Barra, R., Ocampo-Duque, W., Páez, M., Darbra, R.M., Eljarrat, E., Barceló, D., 2013. Occurrence of hydrophobic organic pollutants (BFRs and UV-filters) in sediments from South America. Chemosphere 92, 309-316.

doi:10.1016/j.chemosphere.2013.03.032

Benedé, J.L., Chisvert, A., Giokas, D.L., Salvador, A., 2014a. Development of stir bar sorptive-dispersive microextraction mediated by magnetic nanoparticles and its analytical application to the determination of hydrophobic organic compounds in aqueous media. J. Chromatogr. A 1362, 25-33. doi:10.1016/j.chroma.2014.08.024

Benedé, J.L., Chisvert, A., Salvador, A., Sánchez-Quiles, D., Tovar-Sánchez, A., 2014b. Determination of UV filters in both soluble and particulate fractions of seawaters by dispersive liquid-liquid microextraction followed by gas chromatography-mass 
spectrometry. Anal. Chim. Acta 812, 50-58. doi:10.1016/j.aca.2013.12.033

Blüthgen, N., Zucchi, S., Fent, K., 2012. Effects of the UV filter benzophenone-3 (oxybenzone) at low concentrations in zebrafish (Danio rerio). Toxicol. Appl. Pharmacol. 263, 184-194. doi:10.1016/j.taap.2012.06.008

Botta, C., Labille, J., Auffan, M., Borschneck, D., Miche, H., Cabié, M., Masion, A., Rose, J., Bottero, J.-Y., 2011. TiO2-based nanoparticles released in water from commercialized sunscreens in a life-cycle perspective: Structures and quantities. Environ. Pollut. 159, 1543-1550. doi:10.1016/j.envpol.2011.03.003

Bratkovics, S., Sapozhnikova, Y., 2011. Determination of seven commonly used organic UV filters in fresh and saline waters by liquid chromatography-tandem mass spectrometry. Anal. Methods 3, 2943-2950. doi:10.1039/c1ay05390f

Buser, H.-R., Müller, M.D., Balmer, M.E., Poiger, T., Buerge, I.J., 2005. Stereoisomer Composition of the Chiral UV Filter 4-Methylbenzylidene Camphor in Environmental Samples. Env. Sci Technol 39, 3013-3019. doi:10.1021/es048265r

Chen, J., Dong, X., Xin, Y., Zhao, M., 2011. Effects of titanium dioxide nano-particles on growth and some histological parameters of zebrafish (Danio rerio) after a long-term exposure. Aquat. Toxicol. 101, 493-499. doi:10.1016/j.aquatox.2010.12.004

Chen, L., Hu, J.Y., Wang, S.Q., 2012. The role of antioxidants in photoprotection: a critical review. J. Am. Acad. Dermatol. 67, 1013-1024.

Chen, L.L., Wang, S.Q., 2012. From the bottle to the skin: Challenges in evaluating antioxidants. Photodermatol. Photoimmunol. Photomed. 28, 228-234.

doi:10.1111/j.1600-0781.2012.00674.x

Chisvert, A., Salvador, A., 2007. UV Filters in Sunscreens and other Cosmetics.

Regulatory Aspects and Analytical Methods (3.1), in: Analysis of Cosmetic Products. Elsevier Science, Amsterdam, Holland, pp. 83-120.

Cirer-Costa, J.C., 2012. The beginnings of tourism in Majorca. 1837-1914. Ann. Tour. Res. 39, 1779-1796. doi:10.1016/j.annals.2012.06.004

Cuderman, P., Heath, E., 2007. Determination of UV filters and antimicrobial agents in environmental water samples. Anal. Bioanal. Chem. 387, 1343-1350.

doi:10.1007/s00216-006-0927-y

Danovaro, R., Bongiorni, L., Corinaldesi, C., Giovannelli, D., Damiani, E., Astolfi, P., Greci, L., Pusceddu, A., 2008. Sunscreens Cause Coral Bleaching by Promoting Viral Infections. Environ. Health Perspect. 116, 441-447. doi:10.1289/ehp.10966

Danovaro, R., Corinaldesi, C., 2003. Sunscreen products increase virus production through prophage induction in marine bacterioplankton. Microb. Ecol. 45, 109-118. doi:10.1007/s00248-002-1033-0

Danovaro, R., Damiani, E., Corinaldesi, C., 2014. Sunscreen Compositions. US2014170096 (A1).

Davenport, J., Davenport, J.L., 2006. The impact of tourism and personal leisure transport on coastal environments: A review. Estuar. Coast. Shelf Sci. 67, 280-292. 
doi:10.1016/j.ecss.2005.11.026

Díaz-Cruz, M.S., Barceló, D., 2009. Chemical analysis and ecotoxicological effects of organic UV-absorbing compounds in aquatic ecosystems. TrAC Trends Anal. Chem. 28, 708-717. doi:10.1016/j.trac.2009.03.010

Díaz-Cruz, S., Llorca, M., Barceló, D., 2008. Organic UV filters and their photodegradates, metabolites and disinfection by-products in the aquatic environment. TrAC Trends Anal. Chem. 27, 873-887. doi:10.1016/j.trac.2008.08.012

Diffey, B. 1., 2005. Sunscreens and melanoma: the future looks bright. Br. J. Dermatol. 153, 378-381. doi:10.1111/j.1365-2133.2005.06729.x

Dobbinson, S.J., Wakefield, M.A., Jamsen, K.M., Herd, N.L., Spittal, M.J., Lipscomb, J.E., Hill, D.J., 2008. Weekend Sun Protection and Sunburn in Australia: Trends (19872002) and Association with SunSmart Television Advertising. Am. J. Prev. Med. 34, 94-101. doi:10.1016/j.amepre.2007.09.024

EWG 2014, n.d. Nanomaterials in Sunscreen EWG's 2014 Guide to Sunscreens [WWW Document]. URL http://www.ewg.org/2014sunscreen/nanoparticles-in-sunscreen/ (accessed 9.23.14).

Farah K. Ahmed, 2008. Worldwide Regulation of UV Filters: Current Status and Future Trends, in: Clinical Guide to Sunscreens and Photoprotection, Basic and Clinical Dermatology. Informa Healthcare, pp. 65-82.

Farré, M., Barceló, D., 2012. Chapter 1 - Introduction to the Analysis and Risk of Nanomaterials in Environmental and Food Samples, in: Marinella Farré and Damià Barceló (Ed.), Comprehensive Analytical Chemistry, Analysis and Risk of Nanomaterials in Environmental and Food Samples. Elsevier, pp. 1-32.

Fent, K., Kunz, P.Y., Zenker, A., Rapp, M., 2010a. A tentative environmental risk assessment of the UV-filters 3-(4-methylbenzylidene-camphor), 2-ethyl-hexyl-4trimethoxycinnamate, benzophenone-3, benzophenone-4 and 3-benzylidene camphor. Mar. Environ. Res. 69, S4-S6. doi:10.1016/j.marenvres.2009.10.010

Fent, K., Zenker, A., Rapp, M., 2010b. Widespread occurrence of estrogenic UV-filters in aquatic ecosystems in Switzerland. Environ. Pollut. 158, 1817-1824. doi:10.1016/j.envpol.2009.11.005

Forestier, S., Hansenne, I., Petitdemange, D., 1995. Cosmetic composition comprising the combination of a nanopigment of metal oxides and an antioxidant, and use thereof for protecting the skin and the hair. FR2708851 (A1).

Gago-Ferrero, P., Alonso, M.B., Bertozzi, C.P., Marigo, J., Barbosa, L., Cremer, M., Secchi, E.R., Azevedo, A., Lailson-Brito Jr., J., Torres, J.P.M., Malm, O., Eljarrat, E., Díaz-Cruz, M.S., Barceló, D., 2013a. First determination of UV filters in marine mammals. octocrylene levels in Franciscana dolphins. Environ. Sci. Technol. 47, 56195625. doi:10.1021/es400675y

Gago-Ferrero, P., Díaz-Cruz, M.S., Barceló, D., 2011a. Fast pressurized liquid extraction with in-cell purification and analysis by liquid chromatography tandem mass spectrometry for the determination of UV filters and their degradation products in sediments. Anal. Bioanal. Chem. 400, 2195-2204. doi:10.1007/s00216-011-4951-1 
Gago-Ferrero, P., Díaz-Cruz, M.S., Barceló, D., 2011b. Occurrence of multiclass UV filters in treated sewage sludge from wastewater treatment plants. Chemosphere 84, 1158-1165. doi:10.1016/j.chemosphere.2011.04.003

Gago-Ferrero, P., Díaz-Cruz, M.S., Barceló, D., 2012. An overview of UV-absorbing compounds (organic UV filters) in aquatic biota. Anal. Bioanal. Chem. 404, 25972610. doi:10.1007/s00216-012-6067-7

Gago-Ferrero, P., Díaz-Cruz, M.S., Barceló, D., 2013b. Multi-residue method for trace level determination of UV filters in fish based on pressurized liquid extraction and liquid chromatography-quadrupole-linear ion trap-mass spectrometry. J. Chromatogr. A 1286, 93-101. doi:10.1016/j.chroma.2013.02.056

Gago-Ferrero, P., Mastroianni, N., Díaz-Cruz, M.S., Barceló, D., 2013c. Fully automated determination of nine ultraviolet filters and transformation products in natural waters and wastewaters by on-line solid phase extraction-liquid chromatography-tandem mass spectrometry. J. Chromatogr. A 1294, 106-116. doi:10.1016/j.chroma.2013.04.037

Galán, I., Rodríguez-Laso, Á., Díez-Gañán, L., Cámara, E., 2011. Prevalence and correlates of skin cancer risk behaviors in Madrid (Spain). Gac. Sanit. 25, 44-49. doi:10.1016/j.gaceta.2010.07.013

Gao, L., Yuan, T., Zhou, C., Cheng, P., Bai, Q., Ao, J., Wang, W., Zhang, H., 2013. Effects of four commonly used UV filters on the growth, cell viability and oxidative stress responses of the Tetrahymena thermophila. Chemosphere 93, 2507-2513. doi:10.1016/j.chemosphere.2013.09.041

Gao, Q., Garcia-Pichel, F., 2011. Microbial ultraviolet sunscreens. Nat. Rev. Microbiol. 9, 791-802. doi:10.1038/nrmicro2649

Giokas, D.L., Sakkas, V.A., Albanis, T.A., 2004. Determination of residues of UV filters in natural waters by solid-phase extraction coupled to liquid chromatographyphotodiode array detection and gas chromatography-mass spectrometry. J. Chromatogr. A 1026, 289-293. doi:10.1016/j.chroma.2003.10.114

Giokas, D.L., Sakkas, V.A., Albanis, T.A., Lampropoulou, D.A., 2005. Determination of UV-filter residues in bathing waters by liquid chromatography UV-diode array and gas chromatography-mass spectrometry after micelle mediated extraction-solvent back extraction. J. Chromatogr. A 1077, 19-27. doi:10.1016/j.chroma.2005.04.074

Giokas, D.L., Salvador, A., Chisvert, A., 2007. UV filters: From sunscreens to human body and the environment. TrAC Trends Anal. Chem. 26, 360-374.

doi:10.1016/j.trac.2007.02.012

Goksøyr, A., Tollefsen, K.E., Grung, M., Løken, K., Lie, E., Zenker, A., Fent, K., Schlabach, M., Huber, S., 2009. Balsa Raft Crossing the Pacific Finds Low Contaminant Levels. Environ. Sci. Technol. 43, 4783-4790. doi:10.1021/es900154h

Gómez, M.J., Gómez-Ramos, M.M., Agüera, A., Mezcua, M., Herrera, S., FernándezAlba, A.R., 2009. A new gas chromatography/mass spectrometry method for the simultaneous analysis of target and non-target organic contaminants in waters. J. Chromatogr. A 1216, 4071-4082. doi:10.1016/j.chroma.2009.02.085 
Gondikas, A.P., Von Der Kammer, F., Reed, R.B., Wagner, S., Ranville, J.F., Hofmann, T., 2014. Release of TiO2 nanoparticles from sunscreens into surface waters: A oneyear survey at the old danube recreational lake. Environ. Sci. Technol. 48, 5415-5422. doi:10.1021/es405596y

Gormsen, E., 1997. The impact of tourism on coastal areas. GeoJournal 42, 39-54. doi:10.1023/A:1006840622450

Hall, C.M., 2001. Trends in ocean and coastal tourism: the end of the last frontier? Ocean Coast. Manag., Trends in Ocean Industries 44, 601-618. doi:10.1016/S09645691(01)00071-0

Hanson, K.M., Gratton, E., Bardeen, C.J., 2006. Sunscreen enhancement of UV-induced reactive oxygen species in the skin. Free Radic. Biol. Med. 41, 1205-1212. doi:10.1016/j.freeradbiomed.2006.06.011

Holick, M.F., 2004. Sunlight and vitamin D for bone health and prevention of autoimmune diseases, cancers, and cardiovascular disease. Am. J. Clin. Nutr. 80, 1678S-1688S.

http://www.iet.tourspain.es, n.d. Tourspain website; http://www.iet.tourspain.es [WWW Document]. URL http://www.iet.tourspain.es (accessed 1.27.15).

Hund-Rinke, K., Simon, M., 2006. Ecotoxic Effect of Photocatalytic Active Nanoparticles (TiO2) on Algae and Daphnids (8 pp). Environ. Sci. Pollut. Res. 13, 225232. doi:10.1065/espr2006.06.311

Inbaraj, J.J., Bilski, P., Chignell, C.F., 2002. Photophysical and photochemical studies of 2-phenylbenzimidazole and UVB sunscreen 2-phenylbenzimidazole-5-sulfonic acid. Photochem. Photobiol. 75, 107-116.

Jacobasch, C., Völker, C., Giebner, S., Völker, J., Alsenz, H., Potouridis, T., Heidenreich, H., Kayser, G., Oehlmann, J., Oetken, M., 2014. Long-term effects of nanoscaled titanium dioxide on the cladoceran Daphnia magna over six generations. Environ. Pollut. 186, 180-186. doi:10.1016/j.envpol.2013.12.008

Jacobs, J.F., van de Poel, I., Osseweijer, P., 2010. Sunscreens with Titanium Dioxide (TiO2) Nano-Particles: A Societal Experiment. Nanoethics 4, 103-113.

doi:10.1007/s11569-010-0090-y

Jansen, R., Osterwalder, U., Wang, S.Q., Burnett, M., Lim, H.W., $2013 \mathrm{a}$.

Photoprotection: Part II. Sunscreen: Development, efficacy, and controversies. J. Am. Acad. Dermatol. 69, 867.e1-867.e14. doi:10.1016/j.jaad.2013.08.022

Jansen, R., Wang, S.Q., Burnett, M., Osterwalder, U., Lim, H.W., 2013 b.

Photoprotection: Part I. Photoprotection by naturally occurring, physical, and systemic agents. J. Am. Acad. Dermatol. 69, 853.e1-853.e12. doi:10.1016/j.jaad.2013.08.021

Jovanović, B., 2015. Review of titanium dioxide nanoparticle phototoxicity: Developing a phototoxicity ratio to correct the endpoint values of toxicity tests. Environ. Toxicol. Chem. n/a-n/a. doi:10.1002/etc.2891

Jurado, A., Gago-Ferrero, P., Vàzquez-Suñé, E., Carrera, J., Pujades, E., Díaz-Cruz, M.S., Barceló, D., 2014. Urban groundwater contamination by residues of UV filters. J. 
Hazard. Mater. 271, 141-149. doi:10.1016/j.jhazmat.2014.01.036

Kaiser, D., Sieratowicz, A., Zielke, H., Oetken, M., Hollert, H., Oehlmann, J., 2012. Ecotoxicological effect characterisation of widely used organic UV filters. Environ. Pollut. 163, 84-90. doi:10.1016/j.envpol.2011.12.014

Kameda, Y., Kimura, K., Miyazaki, M., 2011. Occurrence and profiles of organic sunblocking agents in surface waters and sediments in Japanese rivers and lakes. Environ. Pollut. 159, 1570-1576. doi:10.1016/j.envpol.2011.02.055

Kamerow, D., 2014. My new year's wish is for an effective sunscreen. BMJ Online 349. doi:10.1136/bmj.g7729

Kasprzyk-Hordern, B., Dinsdale, R.M., Guwy, A.J., 2008. The occurrence of pharmaceuticals, personal care products, endocrine disruptors and illicit drugs in surface water in South Wales, UK. Water Res. 42, 3498-3518.

doi:10.1016/j.watres.2008.04.026

Kawaguchi, M., Ito, R., Honda, H., Endo, N., Okanouchi, N., Saito, K., Seto, Y., Nakazawa, H., 2008. Simultaneous analysis of benzophenone sunscreen compounds in water sample by stir bar sorptive extraction with in situ derivatization and thermal desorption-gas chromatography-mass spectrometry. J. Chromatogr. A 1200, 260-263. doi:10.1016/j.chroma.2008.05.084

Kim, S., Choi, K., 2014. Occurrences, toxicities, and ecological risks of benzophenone3, a common component of organic sunscreen products: A mini-review. Environ. Int. 70, 143-157. doi:10.1016/j.envint.2014.05.015

Knowles, T., Curtis, S., 1999. The market viability of European mass tourist destinations. A post-stagnation life-cycle analysis. Int. J. Tour. Res. 1, 87-96. doi:10.1002/(SICI)1522-1970(199903/04)1:2<87::AID-JTR135>3.0.CO;2-6

Labille, J., Feng, J., Botta, C., Borschneck, D., Sammut, M., Cabie, M., Auffan, M., Rose, J., Bottero, J.-Y., 2010. Aging of TiO2 nanocomposites used in sunscreen. Dispersion and fate of the degradation products in aqueous environment. Environ. Pollut. 158, 3482-3489. doi:10.1016/j.envpol.2010.02.012

Laborda, F., Bolea, E., Jiménez-Lamana, J., 2014. Single Particle Inductively Coupled Plasma Mass Spectrometry: A Powerful Tool for Nanoanalysis. Anal. Chem. 86, 22702278. doi:10.1021/ac402980q

Lambropoulou, D.., Giokas, D.., Sakkas, V.., Albanis, T.., Karayannis, M.., 2002. Gas chromatographic determination of 2-hydroxy-4-methoxybenzophenone and octyldimethyl-p-aminobenzoic acid sunscreen agents in swimming pool and bathing waters by solid-phase microextraction. J. Chromatogr. A 967, 243-253. doi:10.1016/S0021-9673(02)00781-1

Langford, K.H., Thomas, K.V., 2008. Inputs of chemicals from recreational activities into the Norwegian coastal zone. J. Environ. Monit. 10, 894-898. doi:10.1039/B806198J

Lee, S., Bi, X., Reed, R.B., Ranville, J.F., Herckes, P., Westerhoff, P., 2014.

Nanoparticle Size Detection Limits by Single Particle ICP-MS for 40 Elements.

Environ. Sci. Technol. 48, 10291-10300. doi:10.1021/es502422v 
Lesser, M.P., 2006. Oxidative stress in marine environments: biochemistry and physiological ecology. Annu. Rev. Physiol. 68, 253-278.

doi:10.1146/annurev.physiol.68.040104.110001

Lewicka, Z.A., Benedetto, A.F., Benoit, D.N., Yu, W.W., Fortner, J.D., Colvin, V.L., 2011. The structure, composition, and dimensions of $\mathrm{TiO} 2$ and $\mathrm{ZnO}$ nanomaterials in commercial sunscreens. J. Nanoparticle Res. 13, 3607-3617. doi:10.1007/s11051-011$0438-4$

Li, S., Wallis, L.K., Ma, H., Diamond, S.A., 2014. Phototoxicity of TiO2 nanoparticles to a freshwater benthic amphipod: Are benthic systems at risk? Sci. Total Environ. 466467, 800-808. doi:10.1016/j.scitotenv.2013.07.059

Li, W., Ma, Y., Guo, C., Hu, W., Liu, K., Wang, Y., Zhu, T., 2007. Occurrence and behavior of four of the most used sunscreen UV filters in a wastewater reclamation plant. Water Res. 41, 3506-3512. doi:10.1016/j.watres.2007.05.039

López-Heras, I., Madrid, Y., Cámara, C., 2014. Prospects and difficulties in TiO2 nanoparticles analysis in cosmetic and food products using asymmetrical flow fieldflow fractionation hyphenated to inductively coupled plasma mass spectrometry. Talanta 124, 71-78. doi:10.1016/j.talanta.2014.02.029

Luo, Z., Wang, Z., Xu, B., Sarakiotis, I.L., Laing, G.D., Yan, C., 2014. Measurement and characterization of engineered titanium dioxide nanoparticles in the environment. J. Zhejiang Univ. Sci. A 15, 593-605. doi:10.1631/jzus.A1400111

Magi, E., Di Carro, M., Scapolla, C., Nguyen, K.T.N., 2012. Stir bar sorptive extraction and LC-MS/MS for trace analysis of UV filters in different water matrices.

Chromatographia 75, 973-982. doi:10.1007/s10337-012-2202-z

Ma, H., Brennan, A., Diamond, S.A., 2012. Phototoxicity of TiO2 nanoparticles under solar radiation to two aquatic species: Daphnia magna and Japanese medaka. Environ. Toxicol. Chem. 31, 1621-1629. doi:10.1002/etc.1858

Manaia, E.B., Kaminski, R.C.K., Corrêa, M.A., Chiavacci, L.A., 2013. Inorganic UV filters. Braz. J. Pharm. Sci. 49, 201-209. doi:10.1590/S1984-82502013000200002

Mansfield, C.M., Alloy, M.M., Hamilton, J., Verbeck, G.F., Newton, K., Klaine, S.J., Roberts, A.P., 2015. Photo-induced toxicity of titanium dioxide nanoparticles to Daphnia magna under natural sunlight. Chemosphere 120, 206-210.

doi:10.1016/j.chemosphere.2014.06.075

Mantiñán, M.J.P., Solla, X.M.S., 2010. Impact of Tourism on Coastal Towns: From Improvisation to Planification. Open Urban Stud. J. 3, 21-27.

Miles, A., Waller, J., Hiom, S., Swanston, D., 2005. SunSmart? Skin cancer knowledge and preventive behaviour in a British population representative sample. Health Educ.

Res. 20, 579-585. doi:10.1093/her/cyh010

Miller, D., Wiener, E.-M., Turowski, A., Thunig, C., Hoffmann, H., 1999. O/W emulsions for cosmetics products stabilized by alkyl phosphates - rheology and storage tests. Colloids Surf. Physicochem. Eng. Asp. 152, 155-160. doi:10.1016/S09277757(98)00630-X 
Miller, R.J., Lenihan, H.S., Muller, E.B., Tseng, N., Hanna, S.K., Keller, A.A., 2010. Impacts of Metal Oxide Nanoparticles on Marine Phytoplankton. Env. Sci Technol 44, 7329-7334. doi:10.1021/es100247x

Minetto, D., Libralato, G., Volpi Ghirardini, A., 2014. Ecotoxicity of engineered TiO2 nanoparticles to saltwater organisms: An overview. Environ. Int. 66, 18-27. doi:10.1016/j.envint.2014.01.012

Nguyen, K.T.N., Scapolla, C., Di Carro, M., Magi, E., 2011. Rapid and selective determination of UV filters in seawater by liquid chromatography-tandem mass spectrometry combined with stir bar sorptive extraction. Talanta 85, 2375-2384. doi:10.1016/j.talanta.2011.07.085

OECD Tourism Trends and Policies 2010, n.d. Tourism - OECD; http://www.oecd.org/cfe/tourism/oecdtourismtrendspolicies2010.htm [WWW Document]. URL http://www.oecd.org/cfe/tourism/oecdtourismtrendspolicies2010.htm (accessed 8.25.14).

Oliveira, H.M., Segundo, M.A., Lima, J.L.F.C., Miró, M., Cerdà, V., 2010. On-line renewable solid-phase extraction hyphenated to liquid chromatography for the determination of UV filters using bead injection and multisyringe-lab-on-valve approach. J. Chromatogr. A 1217, 3575-3582. doi:10.1016/j.chroma.2010.03.035

Osterwalder, U., Sohn, M., Herzog, B., 2014. Global state of sunscreens.

Photodermatol. Photoimmunol. Photomed. 30, 62-80. doi:10.1111/phpp.12112

Paredes, E., Perez, S., Rodil, R., Quintana, J.B., Beiras, R., 2014. Ecotoxicological evaluation of four UV filters using marine organisms from different trophic levels Isochrysis galbana, Mytilus galloprovincialis, Paracentrotus lividus, and Siriella armata. Chemosphere 104, 44-50. doi:10.1016/j.chemosphere.2013.10.053

Pedrouzo, M., Borrull, F., Marcé, R.M., Pocurull, E., 2009. Ultra-high-performance liquid chromatography-tandem mass spectrometry for determining the presence of eleven personal care products in surface and wastewaters. J. Chromatogr. A 1216, 6994-7000. doi:10.1016/j.chroma.2009.08.039

Pedrouzo, M., Borrull, F., Marcé, R.M., Pocurull, E., 2010. Stir-bar-sorptive extraction and ultra-high-performance liquid chromatography-tandem mass spectrometry for simultaneous analysis of UV filters and antimicrobial agents in water samples. Anal. Bioanal. Chem. 397, 2833-2839.

Piccinno, F., Gottschalk, F., Seeger, S., Nowack, B., 2012. Industrial production quantities and uses of ten engineered nanomaterials in Europe and the world. J. Nanoparticle Res. 14, 1-11. doi:10.1007/s11051-012-1109-9

Pintado-Herrera, M.G., González-Mazo, E., Lara-Martín, P.A., 2013. Environmentally friendly analysis of emerging contaminants by pressurized hot water extraction-stir bar sorptive extraction-derivatization and gas chromatography-mass spectrometry. Anal. Bioanal. Chem. 405, 401-411. doi:10.1007/s00216-012-6453-1

Plagellat, C., Kupper, T., Furrer, R., de Alencastro, L.F., Grandjean, D., Tarradellas, J., 2006. Concentrations and specific loads of UV filters in sewage sludge originating from a monitoring network in Switzerland. Chemosphere 62, 915-925. 
doi:10.1016/j.chemosphere.2005.05.024

Plass, J., Emeis, D., Blümich, B., 2001. 31P nuclear magnetic resonance studies on alkyl phosphate emulsifiers in cosmetic oil-in-water emulsions. J. Surfactants Deterg. 4, 379-384. doi:10.1007/s11743-001-0191-3

Poiger, T., Buser, H.-R., Balmer, M.E., Bergqvist, P.-A., Müller, M.D., 2004.

Occurrence of UV filter compounds from sunscreens in surface waters: regional mass balance in two Swiss lakes. Chemosphere 55, 951-963.

doi:10.1016/j.chemosphere.2004.01.012

Rabalais, N.N., Turner, R.E., Díaz, R.J., Justić, D., 2009. Global change and eutrophication of coastal waters. ICES J. Mar. Sci. J. Cons. 66, 1528-1537. doi:10.1093/icesjms/fsp047

Ricking, M., Schwarzbauer, J., Franke, S., 2003. Molecular markers of anthropogenic activity in sediments of the Havel and Spree Rivers (Germany). Water Res. 37, 26072617. doi:10.1016/S0043-1354(03)00078-2

Rik Roelandts, 2008. History of Photoprotection, in: Clinical Guide to Sunscreens and Photoprotection, Basic and Clinical Dermatology. Informa Healthcare, pp. 1-10.

Rodil, R., Moeder, M., 2008. Development of a simultaneous pressurised-liquid extraction and clean-up procedure for the determination of UV filters in sediments. Anal. Chim. Acta 612, 152-159. doi:10.1016/j.aca.2008.02.030

Rodil, R., Quintana, J.B., López-Mahía, P., Muniategui-Lorenzo, S., Prada-Rodríguez, D., 2009a. Multi-residue analytical method for the determination of emerging pollutants in water by solid-phase extraction and liquid chromatography-tandem mass spectrometry. J. Chromatogr. A 1216, 2958-2969. doi:10.1016/j.chroma.2008.09.041

Rodil, R., Schrader, S., Moeder, M., 2009b. Pressurised membrane-assisted liquid extraction of UV filters from sludge. J. Chromatogr. A 1216, 8851-8858.

doi:10.1016/j.chroma.2009.10.058

Rodil, R., Schrader, S., Moeder, M., 2009c. Non-porous membrane-assisted liquidliquid extraction of UV filter compounds from water samples. J. Chromatogr. A 1216, 4887-4894. doi:10.1016/j.chroma.2009.04.042

Salvador, A., Chisvert, A., 2005. Sunscreen analysis: A critical survey on UV filters determination. Anal. Chim. Acta 537, 1-14. doi:10.1016/j.aca.2005.01.055

Sánchez-Brunete, C., Miguel, E., Albero, B., Tadeo, J.L., 2011. Analysis of salicylate and benzophenone-type UV filters in soils and sediments by simultaneous extraction cleanup and gas chromatography-mass spectrometry. J. Chromatogr. A 1218, 42914298. doi:10.1016/j.chroma.2011.05.030

Sánchez-Quiles, D., Tovar-Sánchez, A., 2014. Sunscreens as a Source of Hydrogen Peroxide Production in Coastal Waters. Environ. Sci. Technol. 48, 9037-9042. doi:10.1021/es5020696

Sánchez-Quiles, D., Tovar-Sánchez, A., Horstkotte, B., 2013. Titanium determination by multisyringe flow injection analysis system and a liquid waveguide capillary cell in solid and liquid environmental samples. Mar. Pollut. Bull. 76, 89-94. 
doi:10.1016/j.marpolbul.2013.09.024

Santos, A.J.M., Miranda, M.S., Esteves da Silva, J.C.G., 2012. The degradation products of UV filters in aqueous and chlorinated aqueous solutions. Water Res. 46, 3167-3176. doi:10.1016/j.watres.2012.03.057

Sieratowicz, A., Kaiser, D., Behr, M., Oetken, M., Oehlmann, J., 2011. Acute and chronic toxicity of four frequently used UV filter substances for Desmodesmus subspicatus and Daphnia magna. J. Environ. Sci. Health Part A Tox. Hazard. Subst. Environ. Eng. 46, 1311-1319. doi:10.1080/10934529.2011.602936

Sobek, A., Bejgarn, S., Rudén, C., Molander, L., Breitholtz, M., 2013. In the shadow of the Cosmetic Directive - Inconsistencies in EU environmental hazard classification requirements for UV-filters. Sci. Total Environ. 461-462, 706-711.

doi:10.1016/j.scitotenv.2013.05.074

Sun, T.Y., Gottschalk, F., Hungerbühler, K., Nowack, B., 2014. Comprehensive probabilistic modelling of environmental emissions of engineered nanomaterials. Environ. Pollut. 185, 69-76. doi:10.1016/j.envpol.2013.10.004

Tarazona, I., Chisvert, A., León, Z., Salvador, A., 2010. Determination of hydroxylated benzophenone UV filters in sea water samples by dispersive liquid-liquid microextraction followed by gas chromatography-mass spectrometry. J. Chromatogr. A 1217, 4771-4778. doi:10.1016/j.chroma.2010.05.047

Tarazona, I., Chisvert, A., Salvador, A., 2014. Development of a gas chromatographymass spectrometry method for the determination of ultraviolet filters in beach sand samples. Anal. Methods 6, 7772-7780. doi:10.1039/C4AY01403K

Tashiro, Y., Kameda, Y., 2013. Concentration of organic sun-blocking agents in seawater of beaches and coral reefs of Okinawa Island, Japan. Mar. Pollut. Bull. 77, 333-340. doi:10.1016/j.marpolbul.2013.09.013

Tovar-Sánchez, A., Sánchez-Quiles, D., Basterretxea, G., Benedé, J.L., Chisvert, A., Salvador, A., Moreno-Garrido, I., Blasco, J., 2013. Sunscreen Products as Emerging Pollutants to Coastal Waters. PLoS ONE 8, e65451. doi:10.1371/journal.pone.0065451

Trenholm, R.A., Vanderford, B.J., Drewes, J.E., Snyder, S.A., 2008. Determination of household chemicals using gas chromatography and liquid chromatography with tandem mass spectrometry. J. Chromatogr. A 1190, 253-262.

doi:10.1016/j.chroma.2008.02.032

Tsui, M.M.P., Leung, H.W., Lam, P.K.S., Murphy, M.B., 2014. Seasonal occurrence, removal efficiencies and preliminary risk assessment of multiple classes of organic UV filters in wastewater treatment plants. Water Res. 53, 58-67.

doi:10.1016/j.watres.2014.01.014

UNWTO World Tourism Barometer;, 2014. UNWTO World Tourism Barometer; http://mkt.unwto.org/en/barometer [WWW Document]. URL http://mkt.unwto.org/en/barometer

Urbach, F., 2001. The historical aspects of sunscreens. J. Photochem. Photobiol. B, ESP Conference on Photoprotection 64, 99-104. doi:10.1016/S1011-1344(01)00202-0 
US EPA National Center for Environmental Assessment, R.T.P.N., Powers, C., n.d. Nanomaterial Case Studies: Nanoscale Titanium Dioxide in Water Treatment and in Topical Sunscreen (Final) [WWW Document]. URL

http://cfpub.epa.gov/ncea/cfm/recordisplay.cfm?deid=230972 (accessed 9.1.14).

Vidal, L., Chisvert, A., Canals, A., Salvador, A., 2010. Ionic liquid-based single-drop microextraction followed by liquid chromatography-ultraviolet spectrophotometry detection to determine typical UV filters in surface water samples. Talanta 81, 549-555. doi:10.1016/j.talanta.2009.12.042

What's Hot around the Globe: Insights on Personal Care Products. Global Service Studies Website; [WWW Document], 2007. URL

http://kr.en.nielsen.com/reports/GlobalServiceStudies.shtml

Wick, A., Fink, G., Ternes, T.A., 2010. Comparison of electrospray ionization and atmospheric pressure chemical ionization for multi-residue analysis of biocides, UVfilters and benzothiazoles in aqueous matrices and activated sludge by liquid chromatography-tandem mass spectrometry. J. Chromatogr. A 1217, 2088-2103. doi:10.1016/j.chroma.2010.01.079

World Tourism Organization UNWTO;, 2011. Tourism Towards 2030 / Global Overview; http://www.eunwto.org/content/w45127/?p=189899a5998f428f99f66ca0e17d2218 [WWW Document]. URL http://www.eunwto.org/content/w45127/?p=189899a5998f428f99f66ca0e17d2218

WWF Global, n.d. Key threats in the Mediterranean region (http://www.mediterranean.panda.org/threats/) [WWW Document]. URL http://www.mediterranean.panda.org/threats/ (accessed 11.2.14).

Zenker, A., Schmutz, H., Fent, K., 2008. Simultaneous trace determination of nine organic UV-absorbing compounds (UV filters) in environmental samples. J. Chromatogr. A 1202, 64-74. doi:10.1016/j.chroma.2008.06.041

Zucchi, S., Blüthgen, N., Ieronimo, A., Fent, K., 2011a. The UV-absorber benzophenone-4 alters transcripts of genes involved in hormonal pathways in zebrafish (Danio rerio) eleuthero-embryos and adult males. Toxicol. Appl. Pharmacol. 250, 137146. doi:10.1016/j.taap.2010.10.001

Zucchi, S., Oggier, D.M., Fent, K., 2011b. Global gene expression profile induced by the UV-filter 2-ethyl-hexyl-4-trimethoxycinnamate (EHMC) in zebrafish (Danio rerio). Environ. Pollut., Nitrogen Deposition, Critical Loads and Biodiversity 159, 3086-3096. doi:10.1016/j.envpol.2011.04.013 
